\title{
NASA's Planned Return to the Moon: Global Access and Anytime Return Requirement Implications on the Lunar Orbit Insertion Burns
}

\author{
Michelle Garn ${ }^{1},{\mathrm{Min} \mathrm{Qu}^{2} \text {, Jonathan Chrone }}^{2}$, Philip Su${ }^{2}$, Chris Karlgaard ${ }^{2}$ \\ ${ }^{I}$ NASA Langley Research Center, Hampton, VA 23681 \\ ${ }^{2}$ Analytical Mechanics Associates Inc, 303 Butler Farm Road, Hampton, VA 23666
}

\begin{abstract}
Lunar orbit insertion LOI is a critical maneuver for any mission going to the Moon. Optimizing the geometry of this maneuver is crucial to the success of the architecture designed to return humans to the Moon. LOI burns necessary to meet current NASA Exploration Constellation architecture requirements for the lunar sortie missions are driven mainly by the requirement for global access and "anytime" return from the lunar surface. This paper begins by describing the Earth-Moon geometry which creates the worst case $\Delta \mathrm{V}$ for both the LOI and the translunar injection (TLI) maneuvers over the full metonic cycle. The trajectory which optimizes the overall $\Delta \mathrm{V}$ performance of the mission is identified, trade studies results covering the entire lunar globe are mapped onto the contour plots, and the effects of loitering in low lunar orbit as a means of reducing the insertion $\Delta \mathrm{V}$ are described. Finally, the lighting conditions on the lunar surface are combined with the LOIand TLI analyses to identify geometries with ideal lighting conditions at sites of interest which minimize the mission $\Delta \mathrm{V}$.
\end{abstract}

\section{Nomenclature}

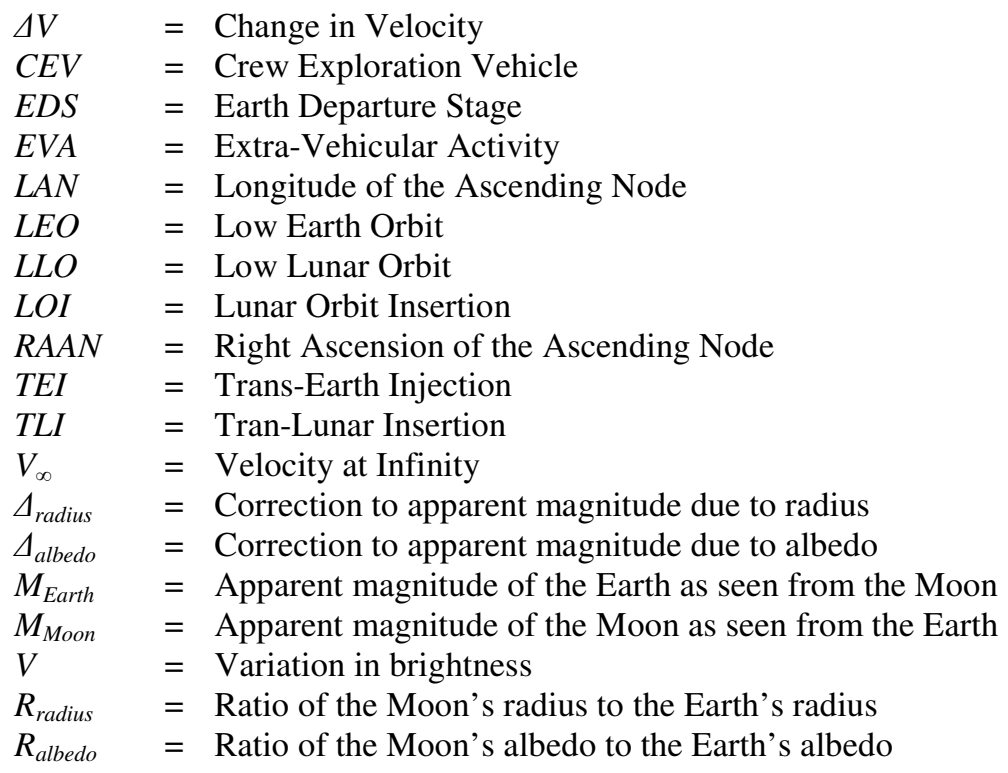




\section{Introduction}

L unar orbit insertion (LOI) is a critical maneuver for any mission going to the Moon. High magnitude $\Delta V$ 's are required to not only allow the spacecraft to be captured by the Moon's gravity well, but also to place the spacecraft into the desired lunar orbit. Optimizing the geometry of this maneuver is crucial to the success of the architecture designed to return humans to the Moon. The LOI burns necessary to meet current NASA Constellation Program architecture requirements for the lunar sortie missions are driven mainly by the requirement for global access and anytime return from the lunar surface. It should be noted that this "global access, anytime return" requirement is very different from that of the Apollo missions,

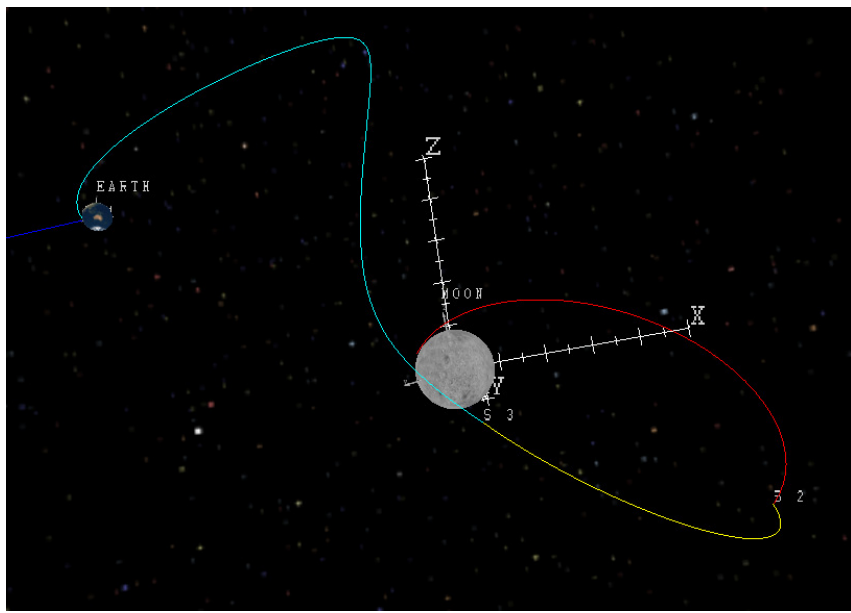

FIGURE 1. Lunar transfer trajectory. Example depiction of a 4 day Earth to Moon transfer followed by the 24 hour 3 burn LOI sequence. which either had landing sites close to the lunar equator or did not require anytime return capability.

For any landing site that is not on the lunar equator or at the poles, the site starts to move away from the initial LLO where the Crew Exploration Vehicle (CEV) is parked. Since the ascent stage is designed to perform only a coplanar ascent, the CEV must perform a nodal plane change to line up with the ascent orbit in the case of "anytime return". To minimize the nodal plane change at LLO, the CEV needs to be placed in a "node walking" orbit such that the wedge angle between the LLO and anytime ascent orbit is small throughout the surface stay. The LLO is uniquely defined depending on the landing site and the surface stay duration. Contrasting to the one-burn maneuver utilized in the Apollo missions, the LOI maneuver is split into 3 burns occurring over a 24-hour period (Figure 1) as most landing sites can become more difficult to reach with a single burn.

Analysis was performed with the location of the second LOI burn constrained to occur at apolune. Subsequent analyses freed the location of the second burn, but required it to be a pure plane change, resulting in a reduced $\Delta \mathrm{V}$. Findings from the analyses also include the need for additional $\Delta \mathrm{V}$ to support missions with a worst case geocentric wedge angle; identification of a method to decrease in $\Delta \mathrm{V}$ by freeing constraints on the LOI timeline, and recommendation of a possible method to further reduce the required $\Delta \mathrm{V}$.

On-going and future work will expand the LOI and TLI data set beyond the worst case epoch to include the entire metonic cycle. This expanded data set will be combined with the corresponding Trans-Earth Injection (TEI) data. This will provide on-orbit mission analyses across the 19-year metonic cycle considering the combined TLI, LOI, and TEI maneuvers.

\section{Methodology and Initial Assumptions}

The focus of this paper is on the outbound portion of the lunar trajectory. Earth departure is from a $28.5^{\circ}$ inclined circular parking orbit at $296 \mathrm{~km}(160 \mathrm{nmi})$ altitude. A 6-hour Earth departure window must be protected in case the nominal departure burn is missed. Lunar arrival is a three-maneuver sequence that puts the vehicle into a circular low-lunar orbit of $100 \mathrm{~km}$ altitude with its inclination and longitude of the ascending node (LAN) tied to a particular landing site for a 7-day surface stay. While the lander takes the crew to the lunar surface, the CEV stays in low-lunar orbit and is capable of performing up to $6.2^{\circ}$ of nodal plane change to line up with the ascent trajectory for rendezvous at anytime during the 7-day stay. The low lunar orbit is also assumed to be retrograde due to a lower propellant requirement in return abort scenarios. The initial outbound mission timeline consists of a 4-day Earth-Moon transfer phase, a 1-day LOI phase and a 1-day orbit checkout phase prior to descent and landing. A more flexible time split between the phases as well as extended post-LOI loitering have also been studied as ways to reduce the overall $\Delta \mathrm{V}$ requirements for the entire mission. 
The Earth-Moon transfers are ballistic-type trajectories modeled in Copernicus, an integrated trajectory design and optimization tool developed at University of Texas, Austin [Ref. 7]. Maneuvers at both Earth departure and lunar arrival are assumed to be impulsive, with Sun, Earth, and Moon gravities included in the modeling. In order to understand the performance requirement for global access, a series of trajectories are optimized in Copernicus targeting low-lunar orbits covering the entire lunar globe set on a $5^{\circ}$ grid in inclination and longitude of the ascending node (LAN). After the worst case epoch is identified, the overall $\Delta \mathrm{V}$ requirements of TLI and LOI are optimized for both the beginning and the end of the Earth departure window, and the results are stored in a database which is then post-processed in Excel. The Excel postprocessor tool can either calculate or optimize the target orbit inclination and LAN based on landing site, stay time and loiter time limit, then evaluate the $\Delta \mathrm{V}$ using a 2-dimensional spline interpolation of the Copernicus database results. A Java based tool called "Astro-Globe" was developed to map the LOI $\Delta \mathrm{V}$ cost onto a lunar globe and allows the user to interactively visualize the correlation between landing site, LLO, loiter, surface duration and $\Delta \mathrm{V}$ in a 3-D environment. Satellite Tool Kit ${ }^{\circledR}$ (STK) is also used to independently spot check the trajectory results generated by Copernicus.

It should be noted that all of the $\Delta \mathrm{V}$ data in this paper are based on impulsive approximation of finite thrust maneuvers and do not account for gravity losses, which are dependent on the vehicle thrust to weight ratio at the beginning of each maneuver and can be added later. The $\Delta \mathrm{V}$ cost for on-orbit plane changes during the LOI maneuver sequence are based on a "fail-safe" arc-type maneuver designed to maintain a safe osculating periapse altitude throughout the burn. Such a maneuver carries a slightly higher $\Delta \mathrm{V}$ cost but provides sufficient time for the flight crew to resolve any propulsion system problems that might interrupt the burn.

\section{Analysis: TLI, LOI, Lighting}

This section will cover the analysis performed in 3 subsections. First the TLI analysis will be covered including discussion of the TLI window frequency, the departure opportunities within a given TLI window, and the effects of the geocentric wedge angle. Second, the LOI analysis will be covered outlining several methods that could be used to reduce LOI $\Delta \mathrm{V}$ and show the results of several of those methods. And finally, lunar lighting analysis including both light from the sun and Earthshine are covered.

\section{A. TLI}

The Earth-Moon transfer is achieved by the Trans-Lunar Injection (TLI) burn from an initial Earth parking orbit of $28.5^{\circ}$ inclination. To minimize the total $\Delta \mathrm{V}$, the TLI maneuver is assumed to be tangential to the velocity direction, which keeps the transfer orbit in-plane with the initial parking orbit. For any desired lunar arrival epoch, the RAAN of the Earth parking orbit and launch time must be chosen such that at the time of arrival the Moon enters the transfer orbit plane. Two possible parking orbits of different RAAN's exist for a given arrival

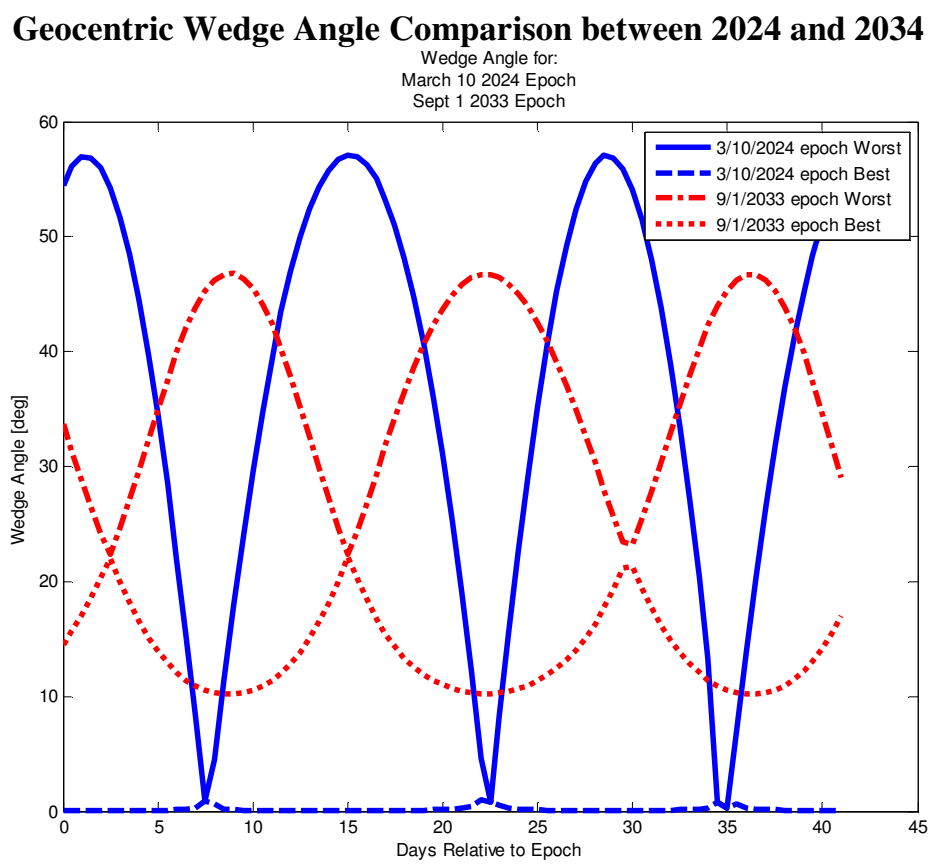

FIGURE 2. Geocentric wedge angle. Could protect for worst case wedge angle in worst year (2024), best case wedge angle in worst year (2034). 
epoch, and one may be more favorable than the other due to the differences in orbital geometry, which may impact the $\Delta \mathrm{V}$ budget as well as mission timeline for later departure opportunities. One of the parameters characterizing this orbital geometry is the geocentric wedge angle between the transfer orbit plane and Moon's orbital plane, which can range anywhere between $0^{\circ}$ and $57^{\circ}$ throughout the metonic cycle. Figure 2 shows how the geocentric wedge angle varies in a typical month in 2024 and 2034. In 2024 the geocentric wedge angle varies between $0^{\circ}$ and $57^{\circ}$ due to Moon's inclination of 28.5 . In 2034 the geocentric wedge angle varies between $10^{\circ}$ and $47^{\circ}$ due to Moon's inclination of $18.5^{\circ}$.

In case the nominal injection burn is missed, the next injection opportunity awaits after the spacecraft makes roughly a full revolution in the parking orbit. The orbital period at a low-Earth orbit is around 90 minutes, which provides five injection opportunities within a 6-hour departure window (Figure $3)$. To ensure that the Moon enters the transfer orbit plane at the time of arrival, the Earth-Moon transfer time would also need to be shortened for subsequent injections opportunities, resulting in higher $\Delta \mathrm{V}$ requirements for both TLI and LOI. In most cases the spacecraft needs to arrive earlier than the nominal arrival time due to the nodal regression of the lowEarth parking orbit. Figure 4 shows how the EarthMoon transfer time varies in subsequent departure opportunities and Figure 5 shows that the EarthMoon transfer time is shortened more dramatically at the end of the 6-hour window when the geocentric wedge angle is small. Initially the Constellation Program planned to protect for up to five departure opportunities, but that has been reduced to two in the most recent studies.

In case TLI is completely missed during the nominal departure window, the next TLI

Earth-Moon Transfer Time Variation (days) within the 6 Hour Departure Window

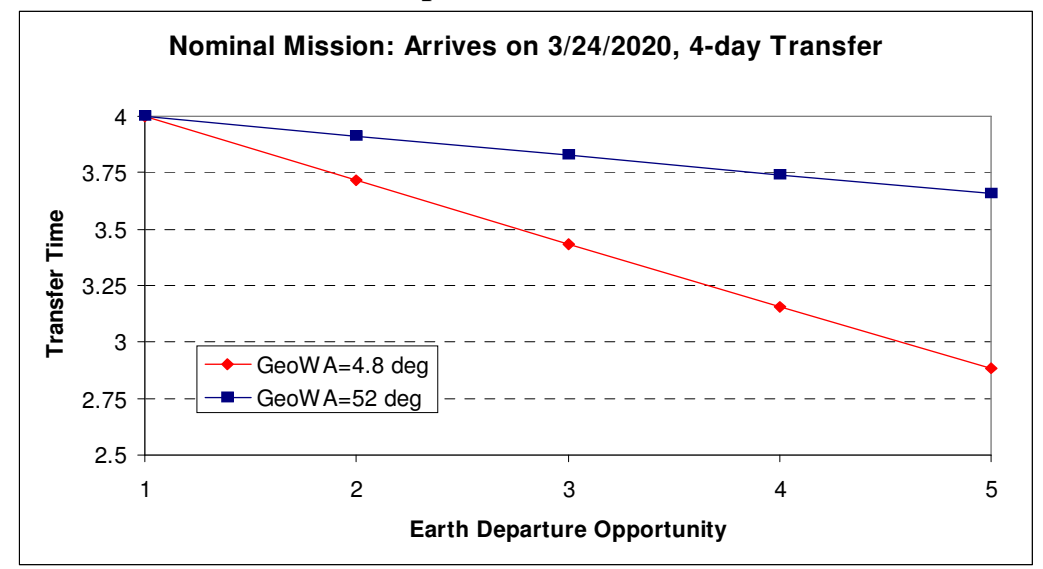

FIGURE 4. TLI departure opportunities.

Geocentric Wedge Angle Effects on Departure Window

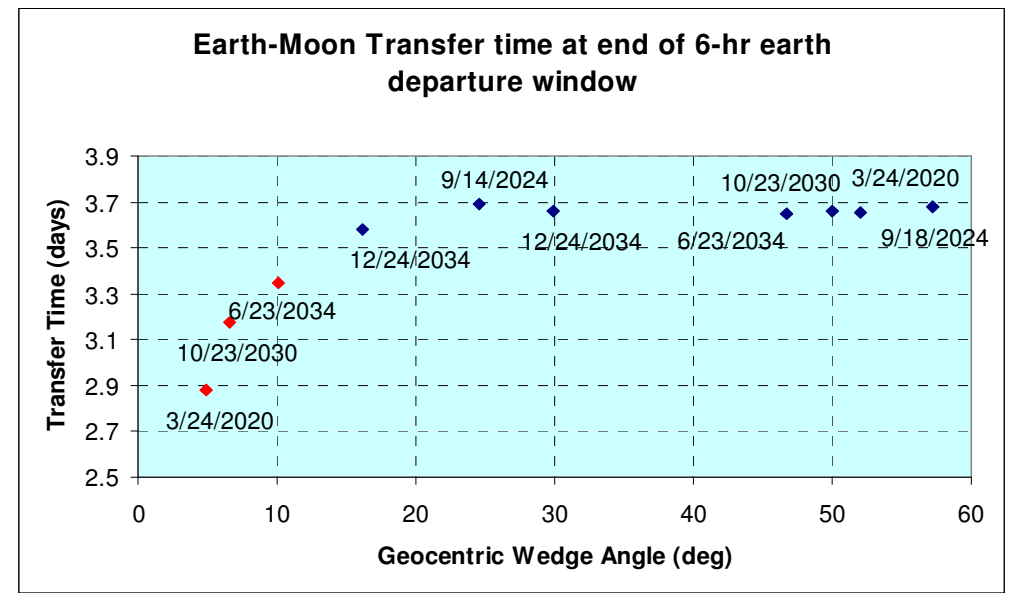

FIGURE 5. TLI departure window. Geocentric wedge angle effects on the TLI transfer time. Reducing transfer time increases $\Delta V$ requirement. 


\section{TLI Opportunities for a Fixed LEO Orbit (Initial Ascending Node $=180$ degrees)}
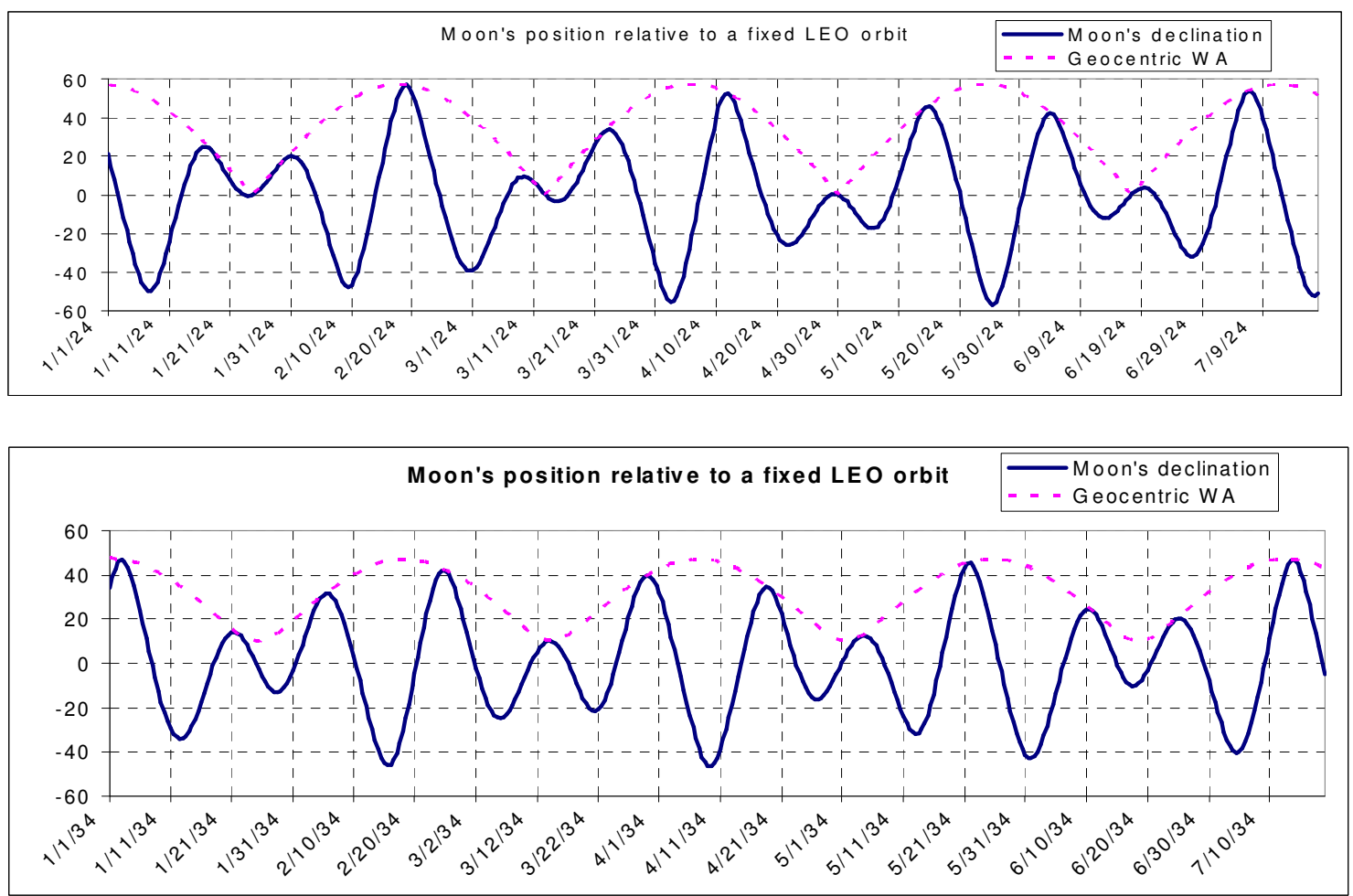

FIGURE 6. TLI opportunities and the associated geocentric wedge angle. The curves shift horizontally as the initial ascending node is changed. Each time the moon's declination crosses zero (blue solid curve) there is a TLI opportunity. Notice in 2034 the opportunities are a little more regularly spaced whereas in 2024 there can be multiple crossing close together. This corresponds to the TLI window opportunities.

departure window arises when the declination of the Moon relative to the parking orbit plane reaches zero at the time of arrival. Figure 6 shows that in 2024 the frequency of such a TLI window can vary anywhere between a few hours to up to around 12 days, while the frequency is much more regularly spaced at 6-10 days in 2034. During periods of the cycle when the geocentric wedge angle was low, opportunities were more frequent, with 1 or 2 days between them, whereas during periods when the wedge angle was high, the intervals between opportunities were less frequent, with 10 or 12 days between opportunities. It is important that the geocentric wedge angle, TLI window, lunar lighting conditions as well as $\Delta \mathrm{V}$ budget all be taken into consideration in selecting the appropriate departure epoch when the mission timeline is being designed.

The main contributing factors to the TLI $\Delta \mathrm{V}$ cost are Earth-Moon transfer time, Earth parking orbit altitude and Moon's distance to Earth at arrival. Everything else being equal, the TLI $\Delta \mathrm{V}$ cost for reaching different parts of the lunar surface does not vary much, which is why most of the data presented in this paper are focused on LOI. However, the geocentric wedge angle, the TLI window and frequency at Earth departure are important aspects of the outbound trajectory and must be studied in detail as they have a significant impact on the performance requirements at lunar arrival.

\section{B. LOI}

The cost of LOI $\Delta \mathrm{V}$ to a given landing site is essentially determined by the $\mathrm{V}_{\infty}$ vector of the hyperbolic orbit coming to the moon and its declination relative to the site-specific targeting "node walking" orbit plane. This relative declination indicates the amount of plane change that must be performed for LOI, which can be as much as 90 degrees for global access to the lunar surface. This section describes the threeburn LOI sequence and methodologies used to reduce $\Delta \mathrm{V}$ requirements, including maneuver optimization and including loiter time. In general, the LOI sequence is a three-burn maneuver to place the CEV/Lunar Lander into a desired circular LLO with specified altitude, inclination and LAN. The desired LLO orbit plane is a function of the landing site latitude and longitude, surface stay time, and maximum on-orbit 
rendezvous plane change allocation in order to meet the any-time return requirements. References 1 and 2 discuss the methodology for calculating the desired LLO based on these inputs.

The cost of overall LOI $\Delta \mathrm{V}$ for global access is determined by the magnitude of the incoming $\mathrm{V}_{\infty}$ vector to the moon. Several factors contribute to the $\mathrm{V}_{\infty}$ vector: transfer time between TLI and lunar arrival (first burn of the LOI), moon's velocity relative to Earth at lunar arrival, and the geocentric wedge angle between the transfer orbit and moon's orbital plane. The worst case arrival epoch has been identified as September 18th of 2024 when the moon is at perigee and in the year when the moon is at its highest inclination of $28.7^{\circ}$. Furthermore, the moon is at nodal crossing relative to Earth's equatorial plane, therefore creating the highest possible geocentric wedge angle of $57.2^{\circ}$, with the assumption that the initial low-Earth orbit (LEO) orbit has an inclination of $28.5^{\circ}$.

The basic LOI sequence is based on a three-burn maneuver sequence to allow for up to $90^{\circ}$ plane change to support global access to the lunar surface. The first burn is an energy-reducing maneuver, the second burn is primarily a plane change maneuver at or near the apolune of the transfer ellipse, and the third burn is a circularization maneuver at a specified altitude. An upper limit of 5 days is placed on the overall flight time, including 1 day for the three-burn LOI sequence. Given these guidelines and LLO targeting information, several methods for generating trajectories can be used. These methods are listed below, in order of complexity:

- Method 1: $2^{\text {nd }}$ LOI burn occurs at apolune, pure plane change

- Method 2: $2^{\text {nd }}$ LOI burn location freed, pure plane change

- Method 3: $2^{\text {nd }}$ LOI burn location freed, arbitrary burn

- Method 4: Split time between 4 days Earth to Moon and 1 day LOI freed (maintain overall 5 days), $2^{\text {nd }}$ LOI burn pure plane change

- Method 5: Split time freed, $2^{\text {nd }}$ LOI burn is arbitrary

The Method 1 approach assumes that the second LOI burn is fixed at apolune and is a pure plane change maneuver. Method 2 frees the location of the second LOI burn to be anywhere along the transfer ellipse but is still constrained to be a pure plane change. Method 3 is a further generalization of Method 2 in which the second LOI burn is arbitrary in purpose (combined plane and energy change) as well as location. Method 4 allows for changes in the flight time between TLI and LOI using a fixed upper bound of 5 days for the total duration, but restricts the second LOI burn to be a pure plane change. Method 5 is the most general method considered in this paper, which also frees the second LOI burn as well as the time split. A sixth method can be envisioned whereby the total transfer time is allowed to vary, but that case is not considered in this paper. In addition, a maximum loiter time in LLO may be considered for each method as a means for reducing the LOI $\Delta \mathrm{V}$.

The various methods are illustrated with the following example. Consider a particular case with a target LLO inclination of $105^{\circ}$ and LAN of $5^{\circ}$, with a departure epoch of September $14^{\text {th }}, 2024,12: 00$ universal time coordinate (UTC) and LLO insertion epoch of September 19 ${ }^{\text {th }}, 2024$ 12:00 UTC (the worst-case epoch). No loiter is considered for this particular case. The results of this example have been calculated using Copernicus and are shown in Table 1. This table shows the $\Delta \mathrm{V}$ required for each maneuver using each of the five methodologies. The parenthetical value for $\Delta \mathrm{V} 2$ shown in the table corresponds to the optimal $\Delta \mathrm{V}$ with additional margin for handling fail-safe plane change maneuvers [Ref 3]. Considerable gains may be found by using the more complicated methods for trajectory optimization with more free variables, as is expected.

Table 1 - Single Case Comparison of Methods 1-5

\begin{tabular}{|l|c|c|c|c|}
\hline & $\mathbf{\Delta V} \mathbf{1}[\mathbf{k m} / \mathbf{s}]$ & $\mathbf{\Delta V} \mathbf{2}[\mathrm{km} / \mathbf{s}]$ & $\mathbf{\Delta V} \mathbf{3}[\mathrm{km} / \mathbf{s}]$ & Total $\mathbf{\Delta V}[\mathrm{km} / \mathbf{s}]$ \\
\hline Method 1 & 0.484 & $0.305(0.334)$ & 0.565 & 1.382 \\
\hline Method 2 & 0.442 & $0.327(0.345)$ & 0.565 & 1.352 \\
\hline Method 3 & 0.375 & $0.372(0.384)$ & 0.565 & 1.324 \\
\hline Method 4 & 0.444 & $0.233(0.242)$ & 0.593 & 1.279 \\
\hline Method 5 & 0.385 & $0.271(0.278)$ & 0.596 & 1.259 \\
\hline
\end{tabular}

Next, these methodologies are applied to a matrix of landing sites corresponding to a $5 \times 5$ degree grid of target orbit inclination and LAN for the worst-case LOI arrival epoch of September 18 ${ }^{\text {th }}, 2024$ 12:00 UTC 
using the worst-case wedge angle. For this study the full lunar surface 5x5 degrees was run for Methods 1 , 2, and 4. Method 3 and 5 were not applied to the full surface access problem due to the additional complication of handling fail-safe plane-change $\Delta \mathrm{V}$ for combined energy and plane change maneuvers.

Method 1 results. The results of applying Method 1 to the full surface access problem are shown in Figures 9-13 in the Appendix. These figures show the total LOI $\Delta \mathrm{V}$ as contours on a latitude vs. longitude global surface scale. Note that the top ten landing sites from the Exploration Systems Architecture Study (ESAS) [Ref. 4] are indicated with the letter symbols. This method assumes a 4 day transfer from TLI to the first LOI burn, a 1 day transfer orbit from the first to third LOI burn, with the second LOI burn consisting of a pure plane change maneuver at the apolune of the transfer orbit. The TLI maneuver is assumed to occur at the beginning of the TLI window. Results for $0,3,5$, and 7 day LLO loiter times are shown. In these results the CEV plane change required for the 7 day lunar sortie global access mission is minimized. The minimum LOI $\Delta \mathrm{V}$ for this method is $0.883 \mathrm{~km} / \mathrm{s}$. With no loiter the maximum LOI $\Delta \mathrm{V}$ is $1.382 \mathrm{~km} / \mathrm{s}$, which exceeds the program requirement of $1.25 \mathrm{~km} / \mathrm{s}$ total $\Delta \mathrm{V}$. For the baseline mission assumption of 3 days of loiter, the maximum $\Delta \mathrm{V}$ is reduced to $1.265 \mathrm{~km} / \mathrm{s}$. This result also exceeds the program requirement of $1.25 \mathrm{~km} / \mathrm{s}$ LOI $\Delta \mathrm{V}$, however, the result can be reduced by using the more sophisticated trajectory optimization methods as will be shown in later sections. The effect of an extended 7 day loiter can reduce the maximum $\Delta \mathrm{V}$ to $1.126 \mathrm{~km} / \mathrm{s}$. Additional loiter can further reduce the total $\Delta \mathrm{V}$ for landing sites such as the Aitken Basin (site B), however, similar results can also be achieved by shortening the Earth-Moon transfer time without adding additional loiter, as is shown in the following sections.

The percent surface coverage is shown as a function of the $\Delta \mathrm{V}$ for the 4-loiter time cases and is calculated based on an area-preserving projection. In the Method 1 result, surface coverage for fixed $\Delta \mathrm{V}$ is only increased by adding loiter time. Other methods shown later can increase surface coverage by varying the Earth-Moon transfer time.

Method 2 results: Figures 14-18 in the Appendix show the results of applying Method 2 optimization to the same epoch for the global surface access analysis. These results are based on the assumption that Earth departure covers the full TLI window. Recall that Method 2 is a generalization of Method 1 in which the location of the second LOI burn is no longer restricted to the apolune of the transfer ellipse, but is still restricted to be pure plane change in nature. The results are shown in the $\Delta \mathrm{V}$ contours on a latitudelongitude plot in Figures 14-17, corresponding to 0, 3, 5, and 7 days of LLO loiter time. In this case, the minimum total $\Delta \mathrm{V}$ is $0.899 \mathrm{~km} / \mathrm{s}$. The maximum $\Delta \mathrm{V}$ for the case with no loiter is $1.354 \mathrm{~km} / \mathrm{s}$, which is a reduction from the Method 1 result but still exceeds the program requirement. Consideration of the baseline 3 day loiter reduces the maximum $\Delta \mathrm{V}$ to $1.243 \mathrm{~km} / \mathrm{s}$, which meets the program requirement. Extended loiter time up to 7 days can decrease the maximum total $\Delta \mathrm{V}$ to $1.099 \mathrm{~km} / \mathrm{s}$.

The percent surface coverage is shown as a function of the $\Delta \mathrm{V}$ for the four loiter time cases. These results show slightly different trends than the results for Method 1 since in this case the full 6 hour TLI window is used rather than the first opportunity.

Method 4 results: In order to further reduce the LOI $\Delta \mathrm{V}$, it is possible to free the Earth-Moon transfer time and include it as an optimization variable, maintaining the overall 5 day total for the transfer time and the three LOI burns. This generalization of Method 2 is known as Method 4 in the nomenclature discussed above. Figures 19-23 in the Appendix show the $\Delta \mathrm{V}$ surface contours for 0,3 , 5, and 7 day loiter times generated using Method 4. In these results the CEV plane change required for the 7-day lunar sortie global access mission is minimized and based on the assumption the Earth departure covers the full TLI window. For the case without loiter, the minimum $\Delta \mathrm{V}$ is $0.893 \mathrm{~km} / \mathrm{s}$ and the maximum $\Delta \mathrm{V}$ is $1.272 \mathrm{~km} / \mathrm{s}$, which is lower than the Method 2 result but still exceeds the program requirements. If 3 days of loiter is added then the maximum $\Delta \mathrm{V}$ is reduced to $1.184 \mathrm{~km} / \mathrm{s}$ which is well below the program requirements and is also substantially lower than the Method 2 result for the same loiter period. Similar trends occur as the loiter time is increased. For the 7 day loiter case, the maximum $\Delta \mathrm{V}$ is reduced to $1.075 \mathrm{~km} / \mathrm{s}$. Figure 24 shows the difference between Method 2 and Method 4 results for the global access case with zero loiter.

For the more costly sites to reach, such as the far side Aitken Basin, freeing the Earth-Moon transfer time allows the spacecraft to reach the site faster in such a way that the total plane change is reduced. For these more costly sites, the rotation of the Moon causes these sites to move away from the orbit plane. Approaching slightly earlier can reduce the magnitude of the plane change requirement on the $2^{\text {nd }}$ LOI burn, which in turn reduces the total LOI $\Delta \mathrm{V}$, keeping the TLI $\Delta \mathrm{V}$ under the program requirements of 3.15 $\mathrm{km} / \mathrm{s}$. Little to no improvement is found for the easier to reach landing sites such as equatorial regions.

Figure 21 shows the percent surface coverage as a function of $\Delta \mathrm{V}$ for the 4 loiter time cases. These results can be directly compared to Figure16 corresponding to Method 2 since both cases protect against 
the full 6 hour TLI window. As expected, the Method 4 results show an improvement in the surface coverage over the Method 2 result.

It should be noted that the LOI $\Delta \mathrm{V}$ results presented in this paper are based on a worst case arrival epoch that results in the highest incoming $\mathrm{V}_{\infty}$ magnitude for a 4-day Earth-Moon transfer. While on any other epoch the overall $\Delta \mathrm{V}$ requirement covering global access is expected to be less than the worst case epoch, it is not guaranteed that this is also the case for every single landing site. Variations in the $\mathrm{V}_{\infty}$ directions can cause the peak site for LOI to shift on different arrival epochs. More thorough studies are ongoing to assess missions covering the entire metonic cycle and to determine both the surface coverage as well as the temporal coverage for any lander design with a certain $\Delta \mathrm{V}$ capability.

\section{Lighting}

Surface lighting conditions are a critical element of mission planning, as they can place additional constraints on mission events such as descent or ascent, as well as on surface activities including extravehicular activities (EVA's).

Solar Lighting: The lighting condition at the landing site is crucial to the planning of surface operations as the lunar night lasts for approximately 14 days over much of the lunar surface and increases dramatically in the polar regions, where night can last for months [Ref 6]. The simplest means of characterizing the surface lighting condition is by analyzing the elevation angle of the sun at the location of interest on the surface. Assuming a spherical Moon and neglecting local terrain, a negative elevation angle indicates the Sun lies below the horizon and that the location is in darkness. Depending on the activity, more specific constraints may be necessary. For example, during landing operations, an elevation angle near $90^{\circ}$ would prevent hazards such as boulders and craters from casting shadows, making them difficult for the crew to detect and avoid. Additionally, constraints on the solar azimuth may also be required. For example, having the sun down range on the lander's trajectory could place the sun directly in the line of sight of the crew, creating a dangerous glare [Ref 6]. Figure 7 shows the results of the solar lighting
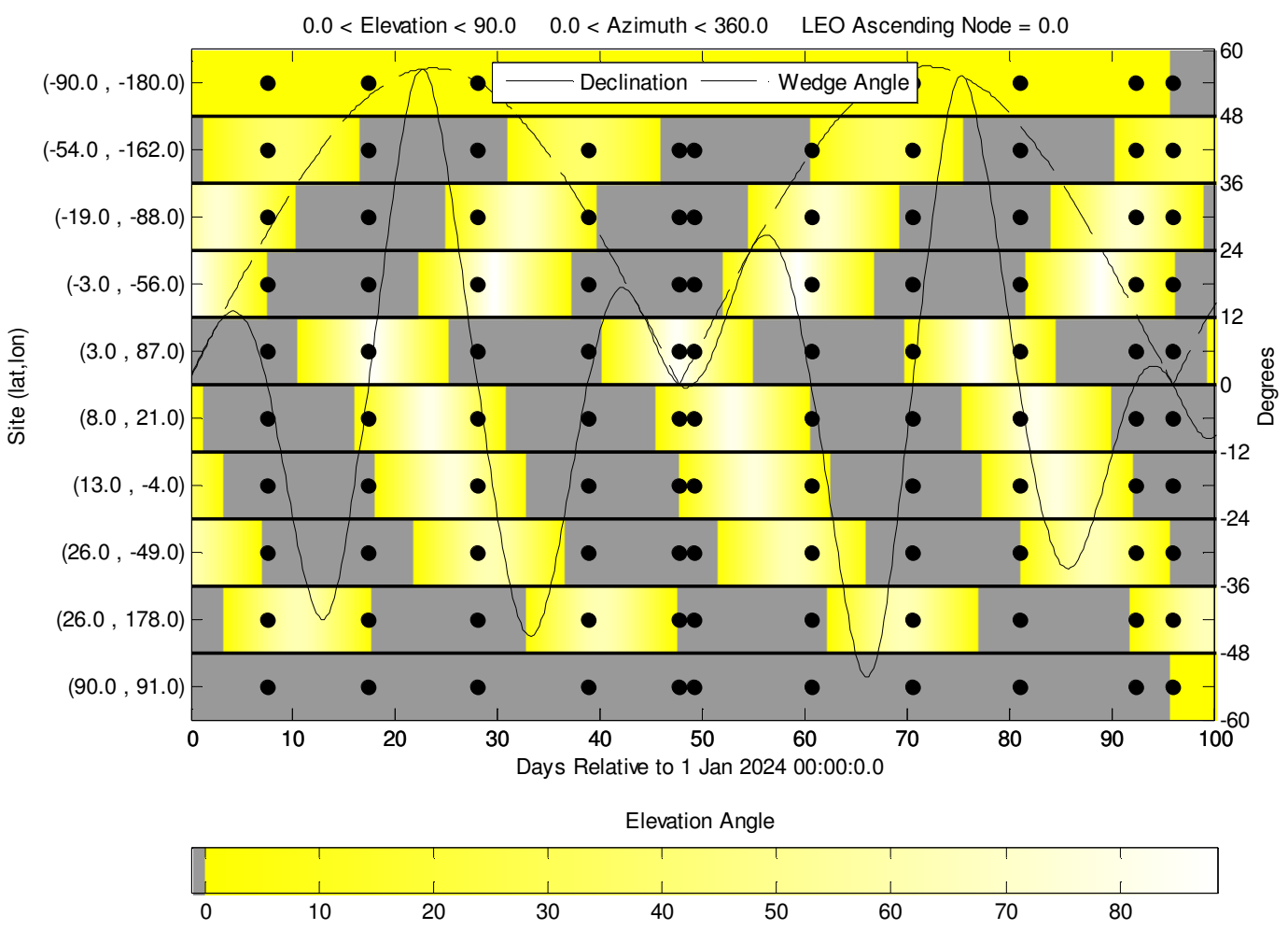

FIGURE 7. Solar lighting conditions on the lunar surface. 
analysis for the 10 ESAS sites, listed on the left hand vertical axis. Each horizontal bar in the figure shows the lighting condition at the corresponding site over time by mapping the solar elevation angle according to the color scale shown at the bottom. Overlayed on top of the lighting data are two curves representing the relative declination of the moon to the LEO at the time of arrival and the magnitude of the geocentric wedge angle. As previously discussed, a value of 0 degrees for this relative declination corresponds to an arrival opportunity. At each time that the declination curve crosses 0 degrees, a column of dots is placed on the figure to indicate the possible arrival times based on the initial ascending node of the LEO.

Earthshine: Light reflected off of the Earth can improve the lighting conditions on the lunar surface during periods of darkness, just as light reflected from the Moon does on Earth, during a full moon in particular. Two angles are required to characterize the effect of Earthshine on the surface lighting condition: the elevation angle of the Earth as viewed from the location of interest and the Sun-Earth-Moon phase angle. Assuming a spherical Moon and neglecting local terrain, a negative elevation angle indicates the Earth lies below the horizon and there cannot be any Earthshine at that location. Values of the phase angle approaching $180^{\circ}$ indicate that the Earth lies almost directly between the Sun and the Moon, preventing light from being reflected off of the Earth to the lunar surface. These two angles are used to create a figure of merit to describe how ideal the Sun-Earth-Moon geometry is for Earthshine at a given
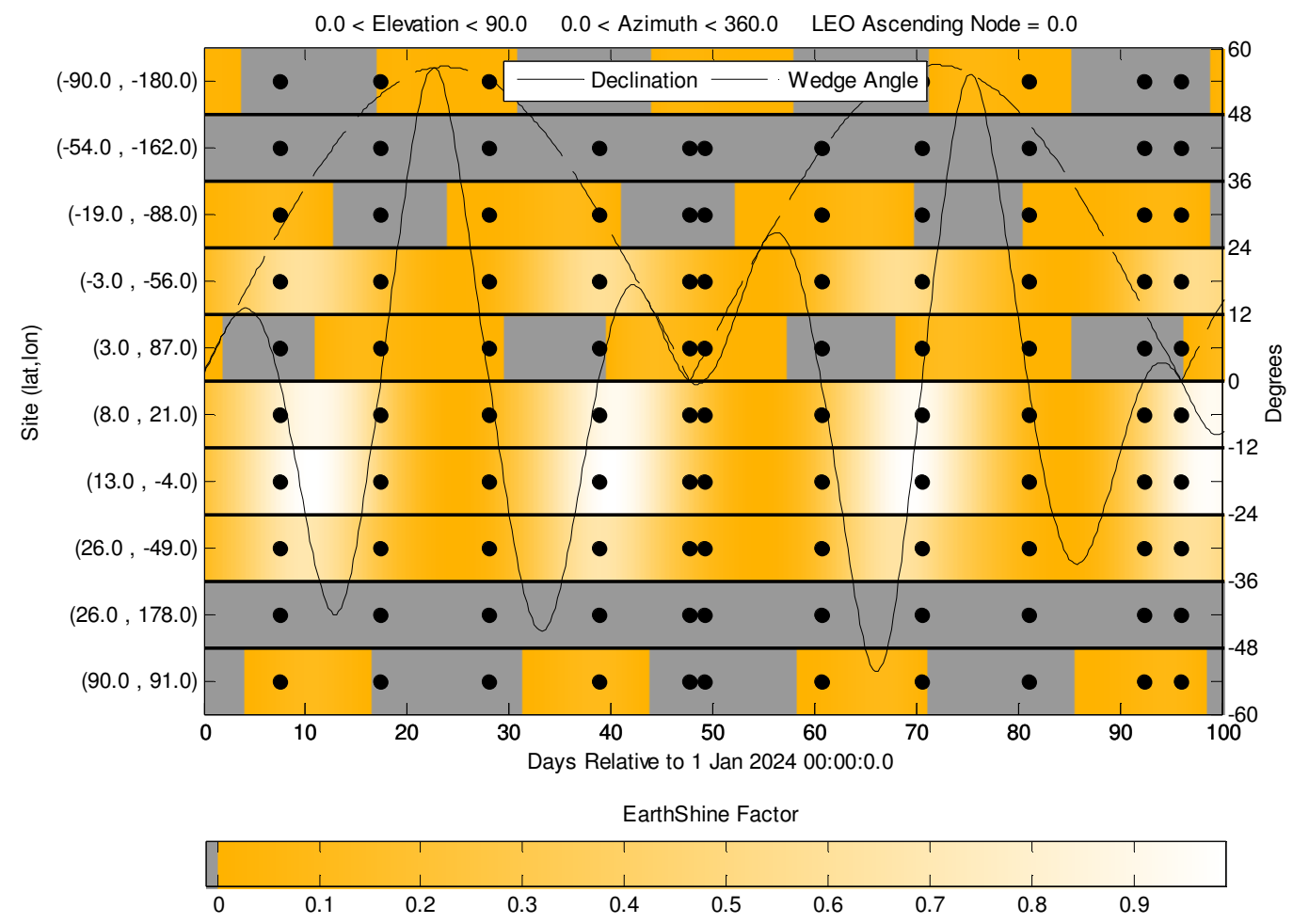

FIGURE 8. Earthshine on the lunar surface.

point in time. Figure 8 shows the results of the Earthshine lighting analysis for the 10 ESAS sites in a format similar to Figure 7. However, rather than showing the elevation angle on the color scale, this figure uses a figure of merit termed 'Earthshine Factor' which describes how well the Sun-Earth-Moon geometry is suited for Earthshine at the location of interest. As previously stated this figure of merit is based on the Sun-Earth-Moon phase angle as well as the elevation angle of the Earth as seen from the location of interest. A value of 0 corresponds to a geometry where Earthshine is impossible, whereas a value of 1 indicates that the geometry is ideally suited for Earthshine. For example, the elevation angle of the Earth as 
seen from a site on the far side of the Moon would always be negative since the Earth lies below the horizon. The Earthshine factor for that site would be 0 .

The brightness of Earthshine under ideal conditions can be approximated based on the apparent magnitude of the full Moon, the ratio of the radii of the Moon and Earth, and the ratio of the albedos of the Moon and Earth. If the Earth and Moon had identical radii and albedo ratios, full Earthshine on the Moon would appear just as a full Moon appears on the Earth and provide the same lighting characteristics. However, the Earth is much larger than the Moon. So, when viewed from the lunar surface the Earth will have a larger angular size. Additionally, the average albedo of the Earth is greater than that of the Moon so more light will be reflected from the Earth to the Moon. Starting with the apparent magnitude of a full Moon as $\mathrm{M}_{\text {Moon }}=-12.55$, the ratio of the Moon's and Earth's radii as $\mathrm{R}_{\text {radius }}=0.272$, and the ratio of the Moon's and Earth's albedos as $\mathrm{R}_{\text {albedo }}=0.324$ the apparent magnitude of the full Earth as seen from the lunar surface can be determined as follows

$$
\begin{gathered}
\Delta_{\text {radius }}=2.5 \log \left(R_{\text {radius }}{ }^{2}\right)=-2.827 \\
\Delta_{\text {albedo }}=2.5 \log \left(R_{\text {albedo }}\right)=-1.223 \\
M_{\text {Earth }}=M_{\text {Moon }}+\Delta_{\text {radius }}+\Delta_{\text {albedo }}=-16.600
\end{gathered}
$$

The difference in brightness, V, of the full Earth and full Moon can be determined based on the apparent magnitudes as follows

$$
V=2.5^{\left(M_{\text {Moon }}-M_{\text {Earh }}\right)}=40.89
$$

So under ideal conditions Earthshine will appear approximately 40 times brighter than a full Moon. It should be noted that this approximation is sensitive to the ratio of the Moon and Earth's albedo. The Moon's albedo is constant and approximately 0.12, whereas the Earth's albedo varies based on weather conditions and surface type. For example, the albedo of clouds, snow, or water can be as high as 0.8 as compared to the average Earth albedo used in this calculation of 0.37. If the calculation is redone using an Earth albedo of 0.8 , the factor changes from approximately 40 to closer to 90 .

\section{Summary of Results}

For the TLI with the initial Earth parking orbit of $28.5^{\circ}$ inclination, two possible parking orbits of different RAAN's exist for a given arrival epoch, and one may be more favorable than the other due to the differences in orbital geometry. The geocentric wedge angle between the parking orbit plane and the Moon's orbital plane can range anywhere between $0^{\circ}$ and $57^{\circ}$ throughout the metonic cycle. There are five injection opportunities within a 6-hour TLI departure window. If the nominal injection burn is missed, the Earth-Moon transfer time would need to be shortened for subsequent injection opportunities, resulting in higher $\Delta \mathrm{V}$ requirements for both TLI and LOI. The transfer time drops more dramatically at the end of the 6-hour window when the geocentric wedge angle is small. Initially the Constellation Program planned to protect for up to five departure opportunities, but that has been reduced to two in the most recent studies in order to reduce propellant requirements. In case the TLI departure window is completely missed, the next TLI departure window arises in different frequencies throughout the metonic cycle.

The LOI sequence is based on a three-burn maneuver sequence to allow for up to $90^{\circ}$ plane change to support global access to the lunar surface. The first burn is an energy-reducing maneuver, the second burn is primarily a plane change maneuver at or near the apolune of the transfer ellipse, and the third burn is a circularization maneuver at a specified altitude. An upper limit of 5 days is placed on the overall flight time, including 1 day for the three-burn LOI sequence. Five methods for generating trajectories are used in order of complexity. The results of these five methods are presented for the worst-case arrival epoch of September $18^{\text {th }}, 202412: 00$ UTC. Then, these methodologies are applied to a matrix of landing sites corresponding to a $5 \times 5$ degree grid of target orbit inclination and LAN for the same epoch using the worstcase wedge angle. For this study the full lunar surface was run for Methods 1,2, and 4. Method 3 and 5 were not applied to the full surface access problem due to the additional complication of handling fail-safe plane-change $\Delta \mathrm{V}$ for combined energy and plane change maneuvers. The results of applying Method 1 and 2 are based on full surface access, up to 7 days LLO loiter, and the minimized CEV plane change. For Method 1, the minimum LOI $\Delta \mathrm{V}$ is $0.883 \mathrm{~km} / \mathrm{s}$. The maximum LOI $\Delta \mathrm{V}$ is $1.382 \mathrm{~km} / \mathrm{s}$ with no loiter and 
$1.126 \mathrm{~km} / \mathrm{s}$ with 7 days loiter time. For Method 2, the minimum LOI $\Delta \mathrm{V}$ is $0.899 \mathrm{~km} / \mathrm{s}$. The maximum LOI $\Delta \mathrm{V}$ is $1.354 \mathrm{~km} / \mathrm{s}$ with no loiter and $1.243 \mathrm{~km} / \mathrm{s}$ with 3 days loiter time and $1.099 \mathrm{~km} / \mathrm{s}$ with 7 days loiter time. No global surface access analysis was conducted using Method 3 and 5 due to computational intensity inherent to this method. For Method 4, the minimum $\Delta \mathrm{V}$ is $0.893 \mathrm{~km} / \mathrm{s}$ and the maximum $\Delta \mathrm{V}$ is $1.272 \mathrm{~km} / \mathrm{s}$ with no loiter. For the 7 day loiter case, the maximum $\Delta \mathrm{V}$ is reduced to $1.075 \mathrm{~km} / \mathrm{s}$.

Surface lighting conditions are a critical element of mission planning since they can place additional constraints on mission events such as descent or ascent, as well as on surface activities. Solar lighting condition can be characterized by analyzing the elevation angle of the Sun at the location of interest on the surface. A negative elevation angle indicates the Sun lies below the horizon and that the location is in darkness. Depending on the activity, more specific constraints may be necessary. Light reflected off of the Earth can improve the lighting conditions on the lunar surface during periods of darkness, just as light reflected from the Moon does on Earth, during a full Moon in particular. Two angles are required to characterize the effect of Earthshine on the surface lighting condition: the elevation angle of the Earth as viewed from the location of interest and the Sun-Earth-Moon phase angle. A negative elevation angle indicates the Earth lies below the horizon and there cannot be any Earthshine at that location. Values of the phase angle approaching $180^{\circ}$ indicate that the Earth lies almost directly between the Sun and the Moon, preventing light from being reflected off of the Earth to the lunar surface. These two angles were used to create a figure of merit to describe how ideal the Sun-Earth-Moon geometry is for Earthshine at a given point in time. The brightness of Earthshine under ideal conditions can be approximated based on the apparent magnitude of the full Moon, the ratio of the radii of the Moon and Earth, and the ratio of the albedos of the Moon and Earth. Under ideal conditions Earthshine will appear approximately either 40 or 90 times brighter than a full Moon, assuming Moon's albedo is 0.12 and Earth's albedo is 0.37 or 0.8 respectively

\section{Conclusion}

The frequency of TLI opportunities was found to vary over the course of the metonic cycle since it is dependent on the geocentric wedge angle. During periods of the cycle when the geocentric wedge angle is low, opportunities are more frequent, with 1 or 2 days between them, whereas during periods when the wedge angle is high, the intervals between opportunities are less frequent, with 10 or 12 days between opportunities. The geocentric wedge angle was also found to affect the Earth-Moon transfer time for off nominal Earth departures. A nominal 4 day Earth-Moon flight time was found to require a transfer time of $\sim 3.7$ days for a departure at the end of the 6 hour window for large geocentric wedge angles and $\sim 2.8$ days for low wedge angles. The reduced flight time requires a higher magnitude TLI maneuver, potentially increasing the $\Delta \mathrm{V}$ above the TLI requirement and reducing the length of the TLI window.

Loiter in LLO was found to have a significant reduction on the $\Delta \mathrm{V}$ cost for the three-burn LOI maneuver in all methodologies examined, showing 7-10\% reductions with 3 days of loiter and 15-18\% reductions at 7 days. This loiter, successfully reduces the LOI $\Delta \mathrm{V}$ below the program requirement of 1250 $\mathrm{m} / \mathrm{s}$ for complete global access. Method 2 was able to reduce the global maximum $\Delta \mathrm{V}$ while fully protecting for a 6 hour TLI window. Method 4 was able to reduce the $\Delta \mathrm{V}$ further, particularly for difficult to reach sites like the Aitken Basin.

Lighting conditions on the lunar surface were examined at the 10 ESAS sites of interest over an extended period of time. For most of the sites the lunar night was found to last $\sim 14$ days except in regions near the poles where the night extends much longer, $\sim 3$ months. Earthshine was also investigated and under ideal conditions found to provide illumination significantly brighter than the light of a full Moon. 


\section{Appendix}

This section includes the full surface LOI $\Delta$ Vs including loiter cases for methods 1,2 , and 4 .

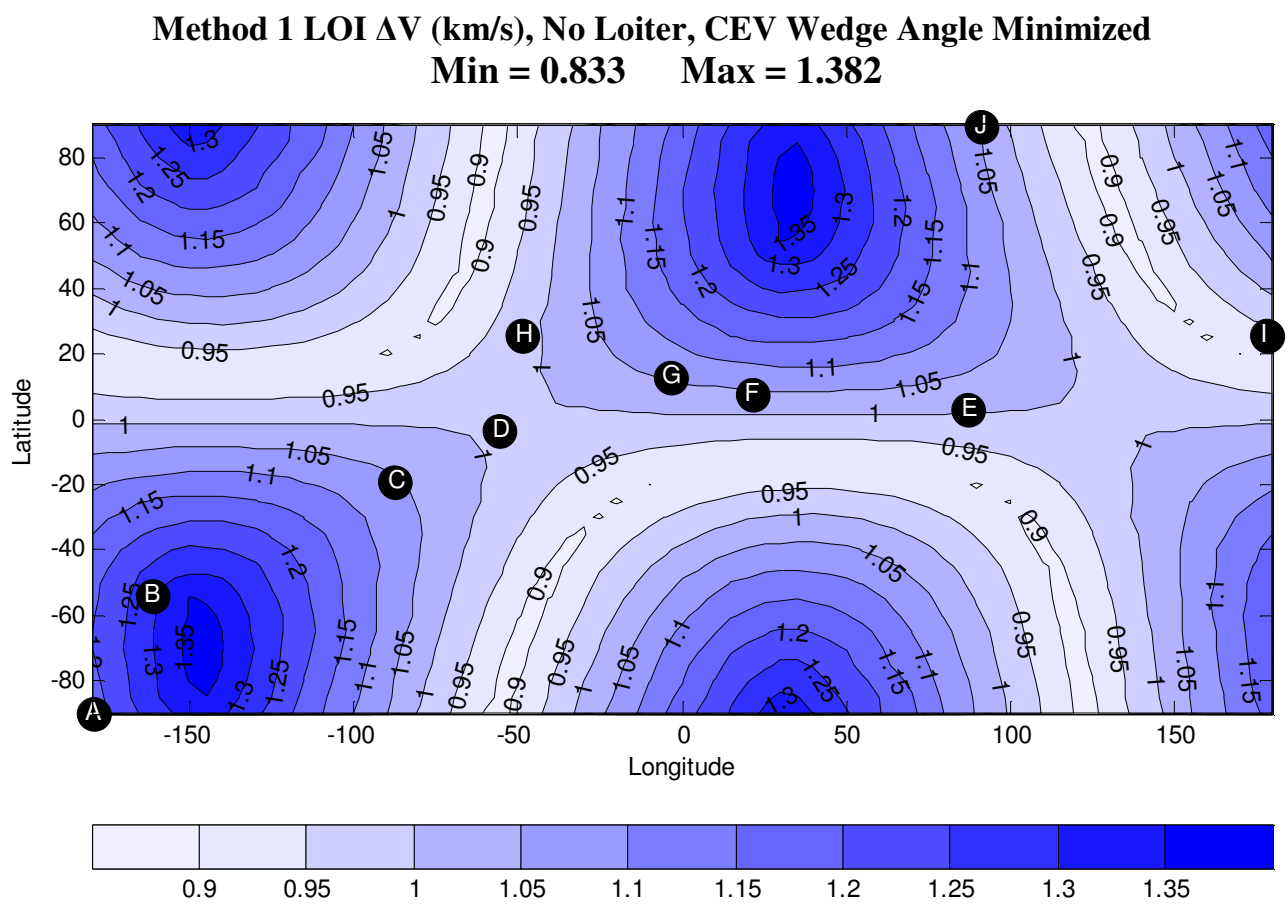

FIGURE 9. Method 1, no loiter. No LLO loiter method 1 four day transfer time from the earth to the moon, 2nd LOI burn at apolune as a pure plane change, nominal mission with earth departure at the beginning of the TLI window using the worst case Epoch for LOI and using the worst case geocentric wedge angle.

Method 1 LOI $\Delta V(\mathrm{~km} / \mathrm{s})$, Loiter Limit = 3 days, CEV Wedge Angle Minimized $\operatorname{Min}=\mathbf{0 . 8 3 3} \quad \operatorname{Max}=\mathbf{1 . 2 6 5}$

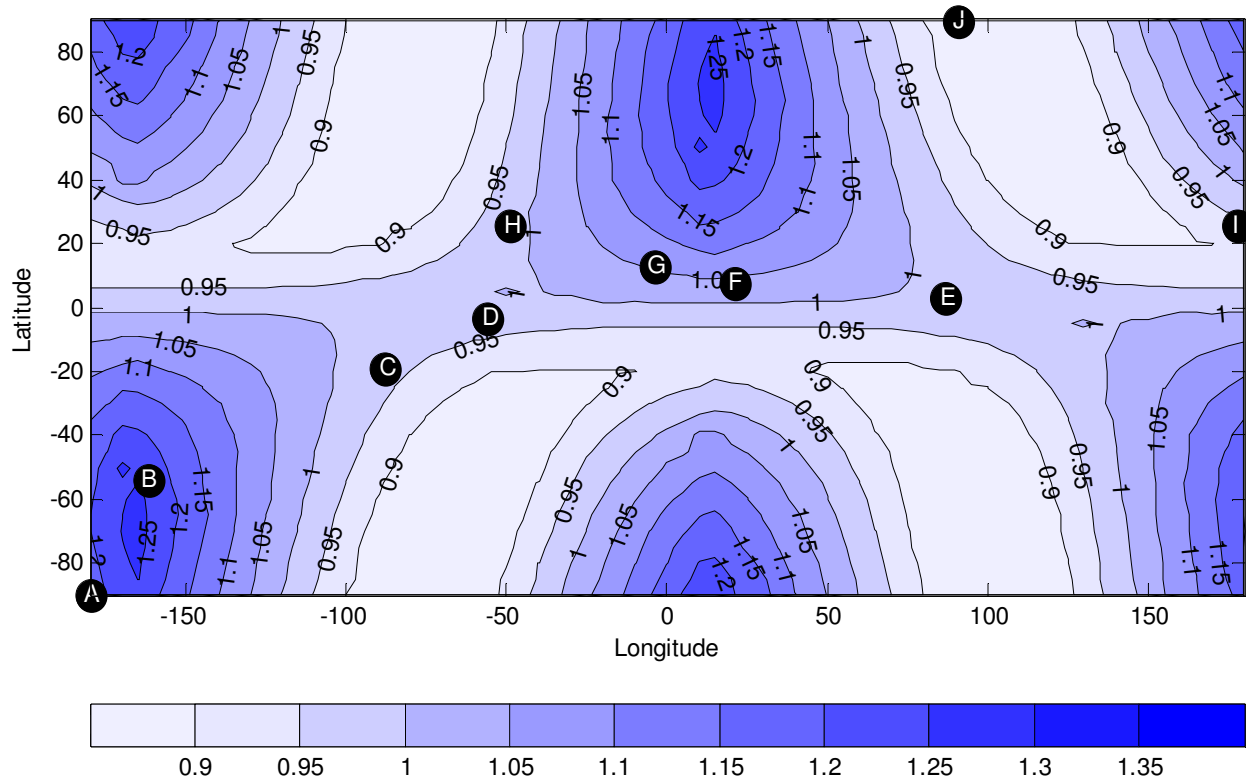

FIGURE 10. Method 1, loiter limit $=3$ days. Up to 3 days of LLO loiter for method 1 four day transfer time from the earth to the moon, 2nd LOI burn at apolune as a pure plane change, nominal mission with earth departure at the beginning of the TLI window using the worst case Epoch for LOI and using the worst case geocentric wedge angle. 
Method 1 LOI $\Delta V(\mathrm{~km} / \mathrm{s})$, Loiter Limit = 5 days, CEV Wedge Angle Minimized $\operatorname{Min}=\mathbf{0 . 8 3 3} \quad \operatorname{Max}=\mathbf{1 . 1 9 8}$

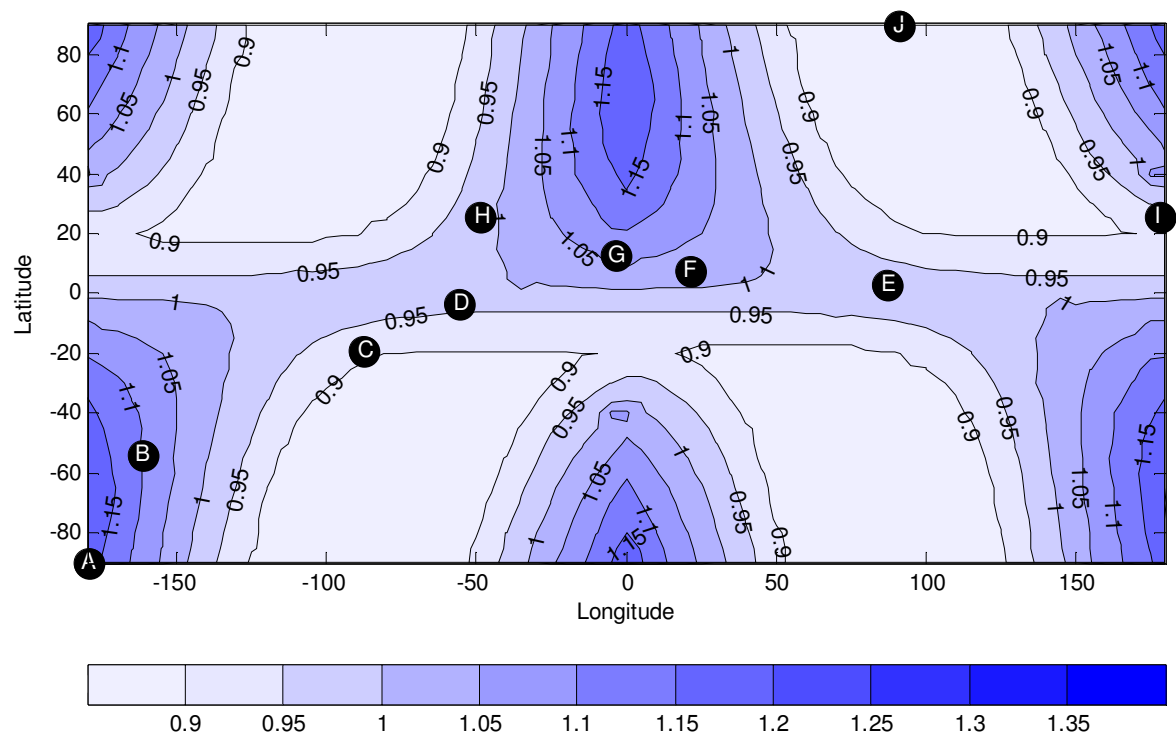

FIGURE 11. Method 1, loiter limit $=5$ days. Up to 5 days of LLO loiter for method 1 four day transfer time from the earth to the moon, 2nd LOI burn at apolune as a pure plane change, nominal mission with earth departure at the beginning of the TLI window using the worst case Epoch for LOI and using the worst case geocentric wedge angle.

\section{Method 1 LOI $\Delta V(\mathrm{~km} / \mathrm{s})$, Loiter Limit = 7 days, CEV Wedge Angle Minimized} $\operatorname{Min}=\mathbf{0 . 8 3 3} \quad \operatorname{Max}=1.126$
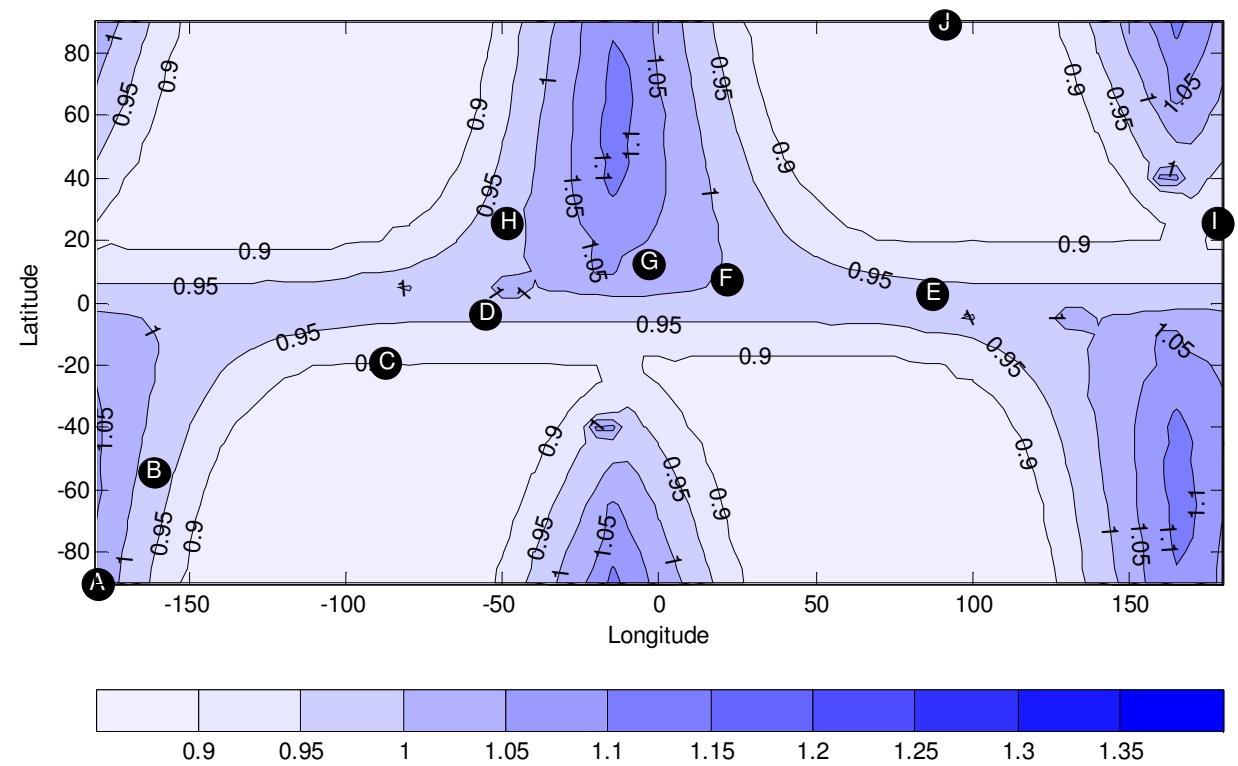

FIGURE 12. Method 1, loiter limit $=7$ days. Up to 7 days of LLO loiter for method 1 four day transfer time from the earth to the moon, 2nd LOI burn at apolune as a pure plane change, nominal mission with earth departure at the beginning of the TLI window using the worst case Epoch for LOI and using the worst case geocentric wedge angle. 


\section{Method 1 Surface Coverage vs. $\Delta$ V Capability}

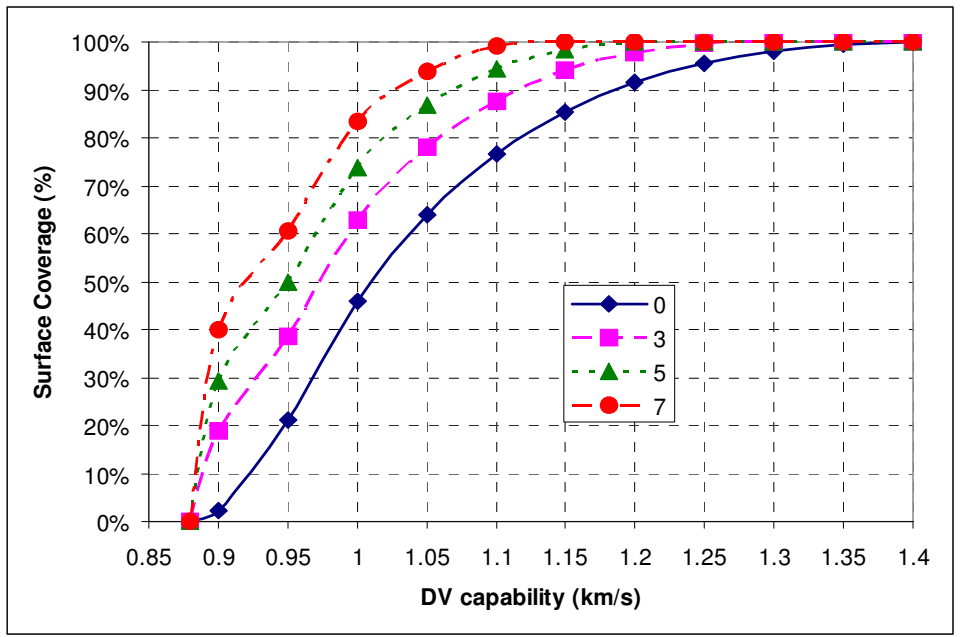

FIGURE 13. Method 1, surface coverage. Percentage of surface coverage for method 1 for different maximum LLO loiter cases.

\section{Method 2 LOI $\Delta V(\mathrm{~km} / \mathrm{s})$, No Loiter, CEV Wedge Angle Minimized} $\operatorname{Min}=\mathbf{0 . 8 9 9} \quad \operatorname{Max}=1.354$
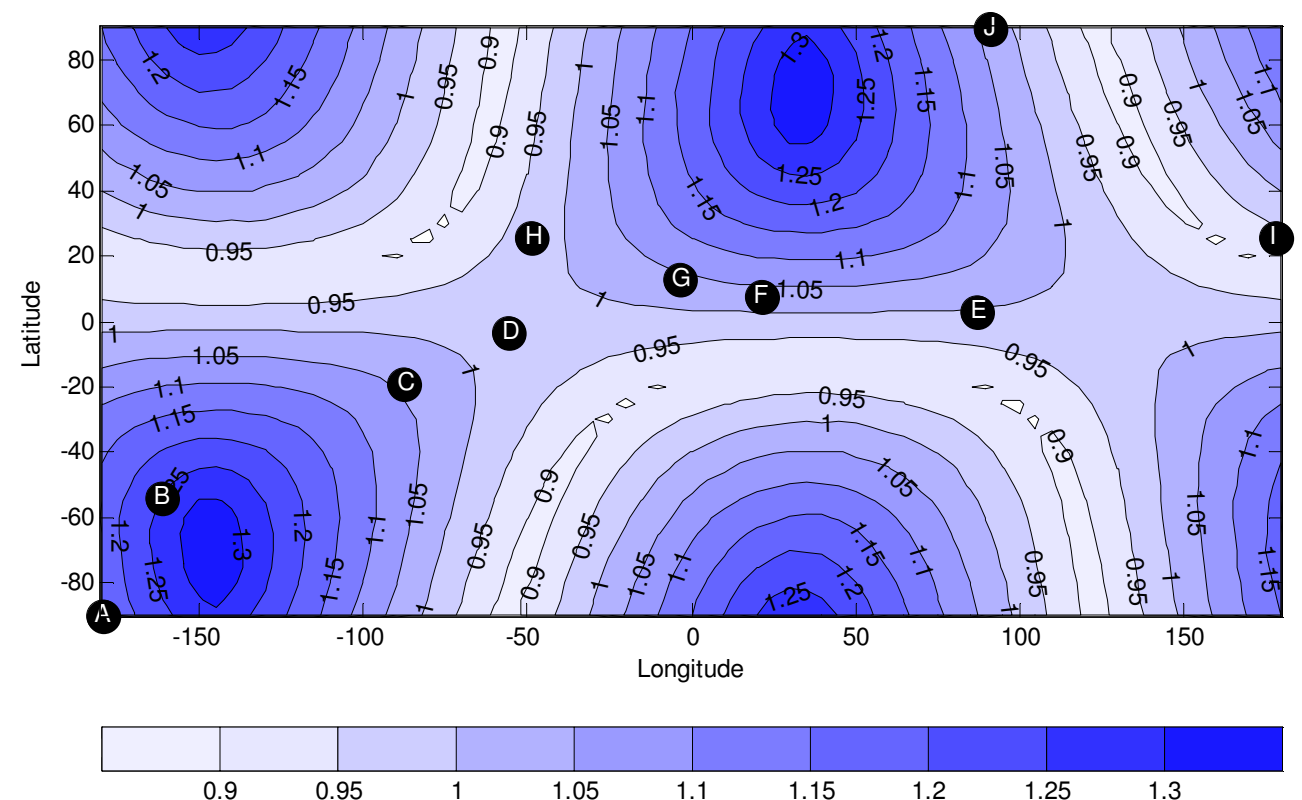

FIGURE 14. Method 2, no loiter. No LLO loiter method 2 four day transfer time from the earth to the moon, 2nd LOI burn time freed, burn is a pure plane change, earth departure covering the full TLI window, using the worst case Epoch for LOI and using the worst case geocentric wedge angle. 
Method 2 LOI $\Delta \mathrm{V}(\mathrm{km} / \mathrm{s})$, Loiter Limit $=3$ Days, CEV Wedge Angle Minimized
Min $=0.885 \quad \operatorname{Max}=1.243$
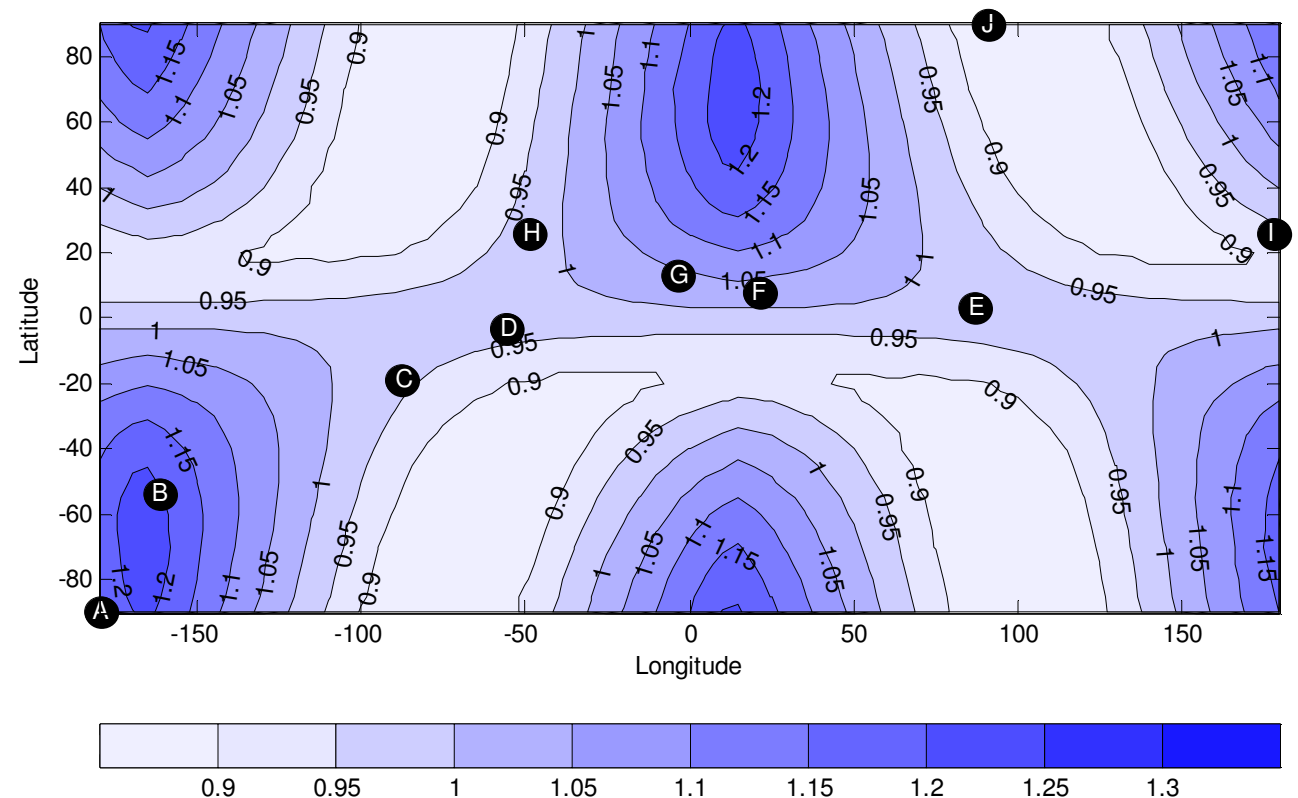

FIGURE 15. Method 2, loiter limit $=3$ days. Up to 3 days LLO loiter method 2 four day transfer time from the earth to the moon, 2nd LOI burn time freed, burn is a pure plane change, earth departure covering the full TLI window, using the worst case Epoch for LOI and using the worst case geocentric wedge angle.

Method 2 LOI $\Delta$ V $(\mathrm{km} / \mathrm{s})$, Loiter Limit = 5 Days, CEV Wedge Angle Minimized $\operatorname{Min}=\mathbf{0 . 8 8 5} \quad \operatorname{Max}=\mathbf{1 . 1 7 1}$
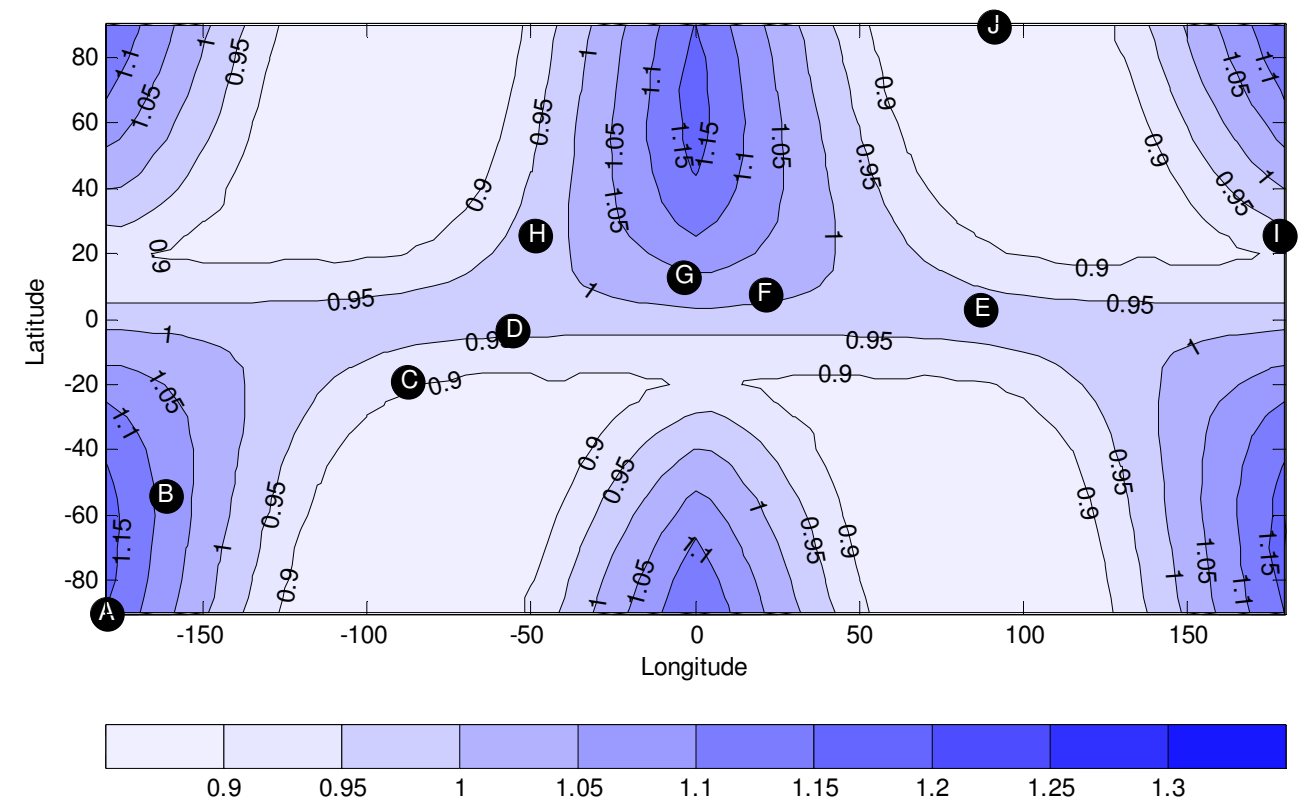

FIGURE 16. Method 2, loiter limit $=5$ days. Up to 5 days LLO loiter method 2 four day transfer time from the earth to the moon, 2nd LOI burn time freed, burn is a pure plane change, earth departure covering the full TLI window, using the worst case Epoch for LOI and using the worst case geocentric wedge angle. 
Method 2 LOI $\Delta V(k m / s)$, Loiter Limit = 7 Days, CEV Wedge Angle Minimized $\operatorname{Min}=0.885 \quad \operatorname{Max}=1.099$
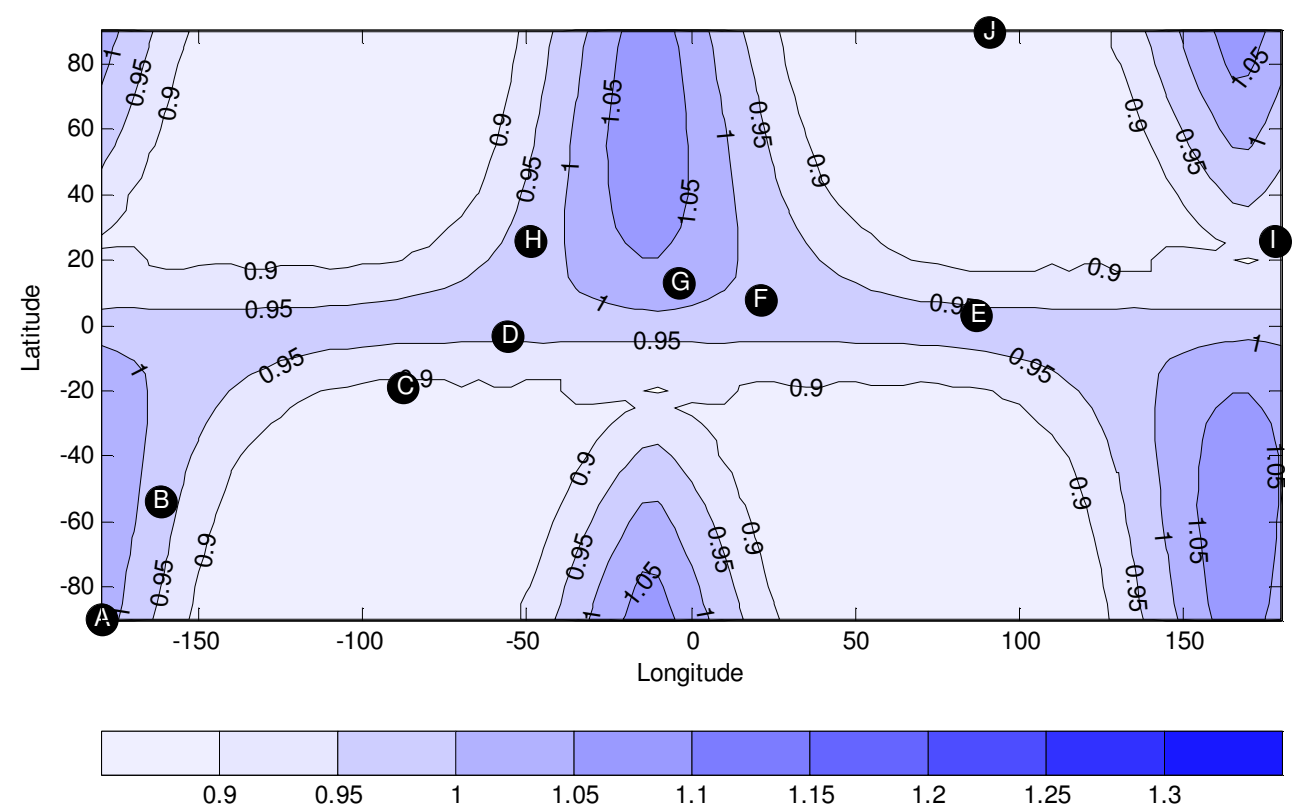

FIGURE 17. Method 2, loiter limit $=7$ days. Up to 7 days LLO loiter method 2 four day transfer time from the earth to the moon, 2nd LOI burn time freed, burn is a pure plane change, earth departure covering the full TLI window, using the worst case Epoch for LOI and using the worst case geocentric wedge angle.

Method 2 Surface Coverage vs. $\Delta$ V Capability

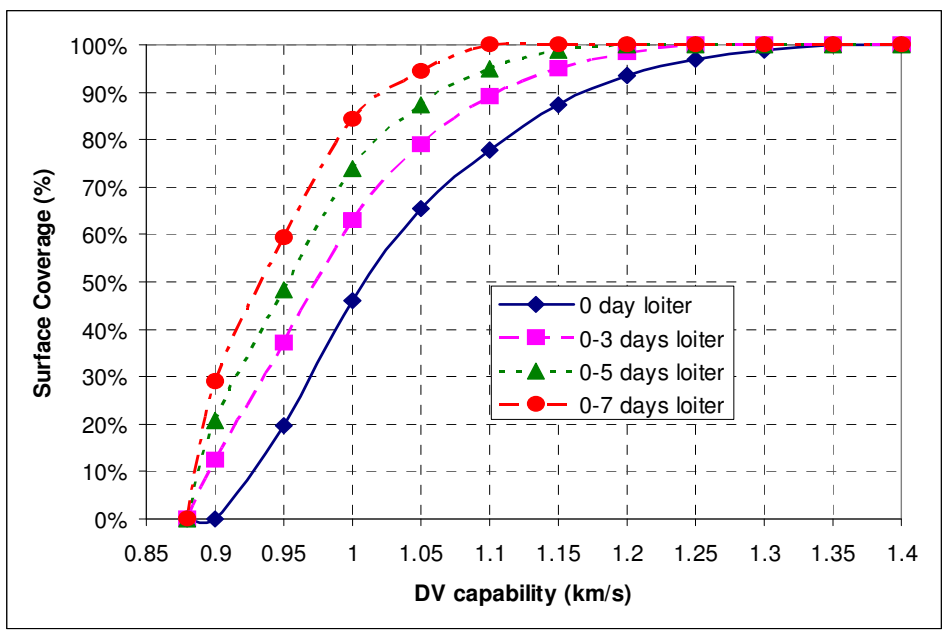

FIGURE 18. Method 2, surface coverage. Percentage of surface coverage for method 2 for different maximum LLO loiter cases. 

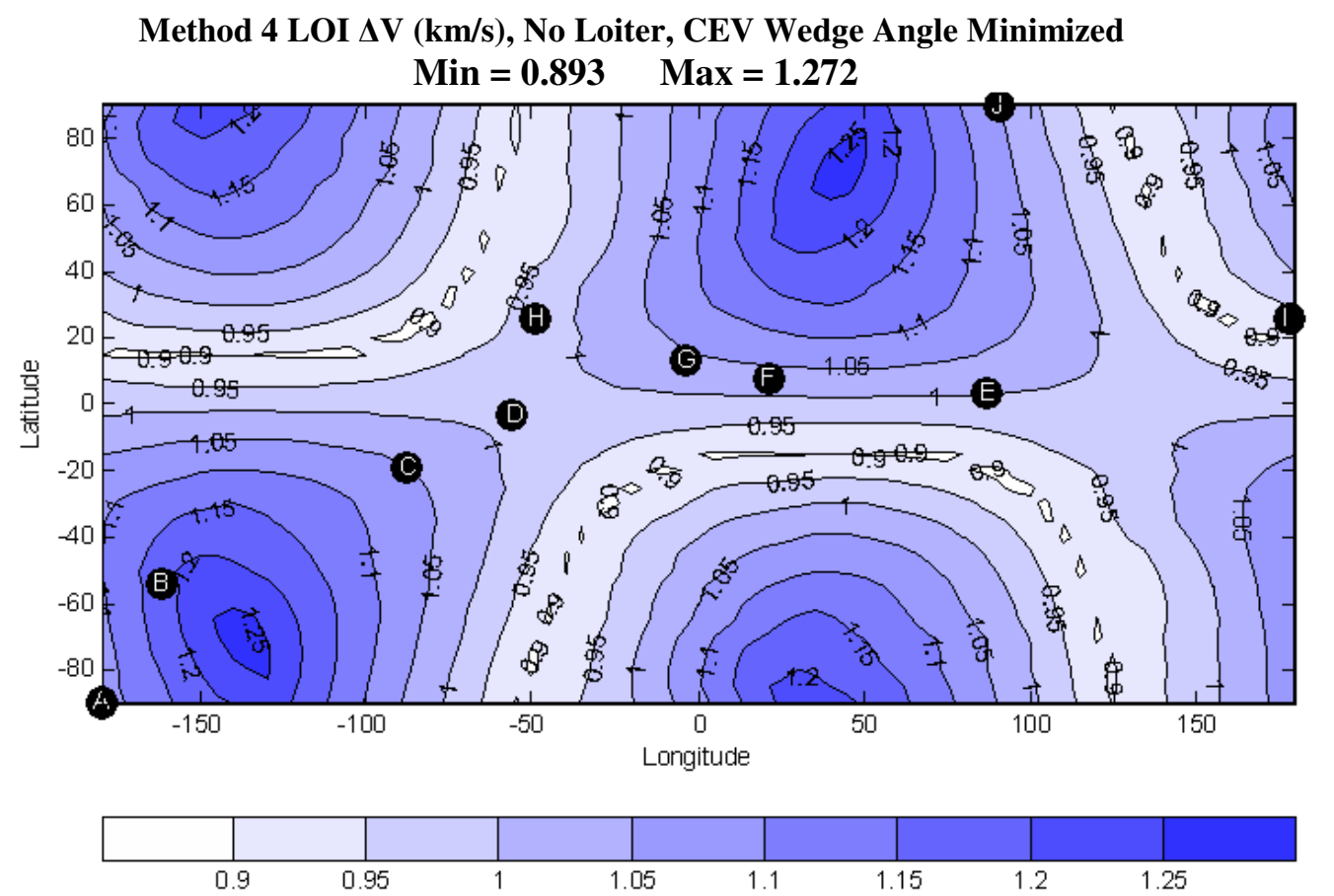

FIGURE 19. Method 4, no loiter. No LLO loiter method 4, transfer time from the earth to the moon freed, 2nd LOI burn time freed, burn is a pure plane change, earth departure covering the full TLI window, using the worst case Epoch for LOI and using the worst case geocentric wedge angle.

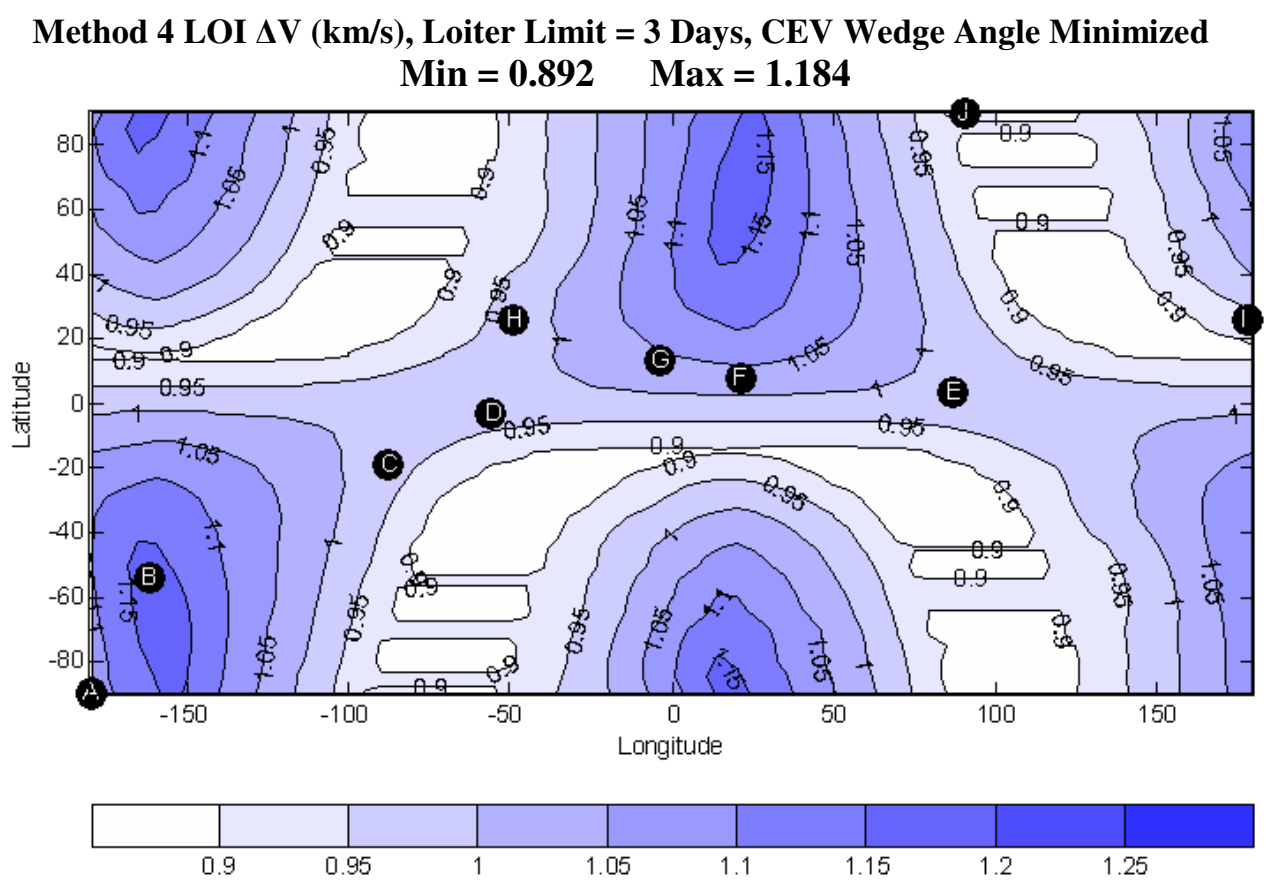

FIGURE 20. Method 4, loiter limit $=3$ days. LLO loiter limit $=3$ days, method 4 , transfer time from the earth to the moon freed, 2nd LOI burn time freed, burn is a pure plane change, earth departure covering the full TLI window, using the worst case Epoch for LOI and using the worst case geocentric wedge angle. 

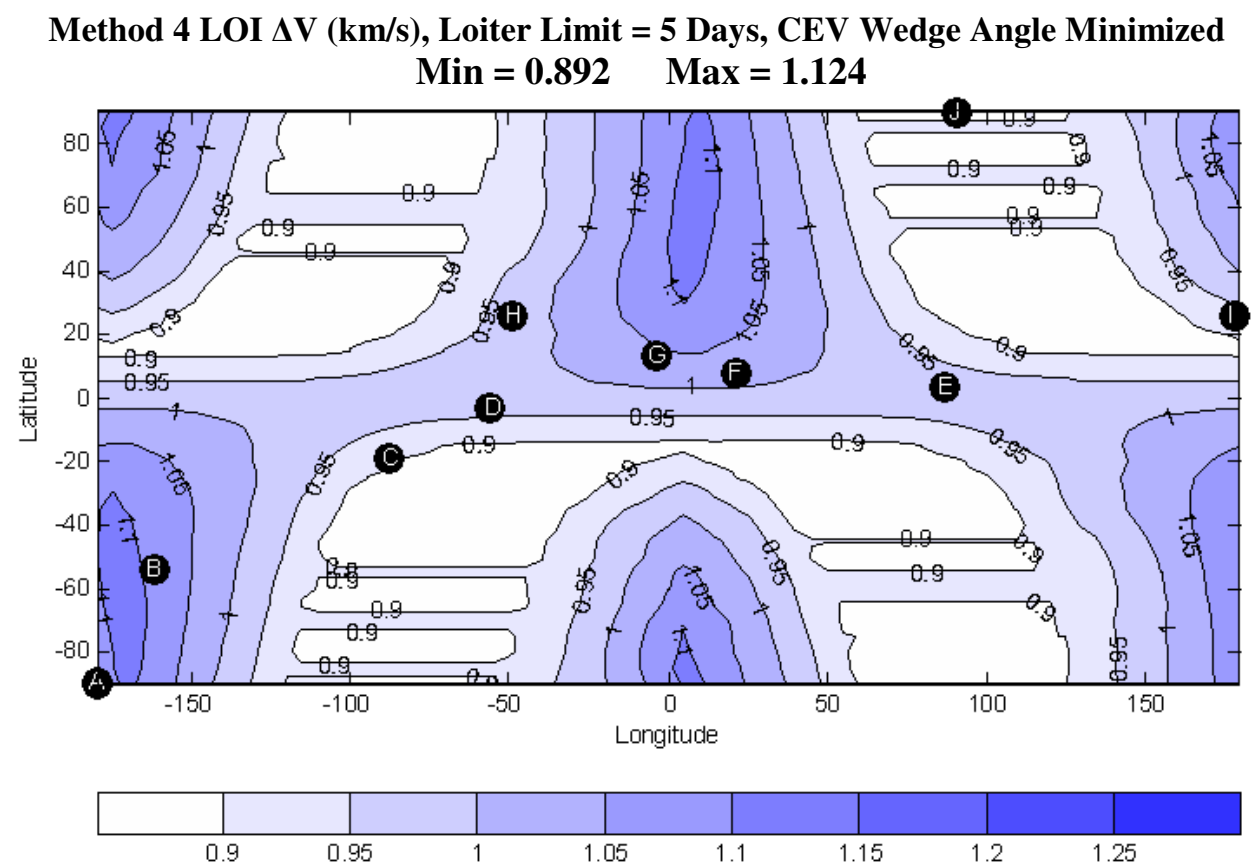

FIGURE 21. Method 4, loiter limit $=5$ days. LLO loiter limit $=5$ days, method 4 , transfer time from the earth to the moon freed, 2nd LOI burn time freed, burn is a pure plane change, earth departure covering the full TLI window, using the worst case Epoch for LOI and using the worst case geocentric wedge angle.

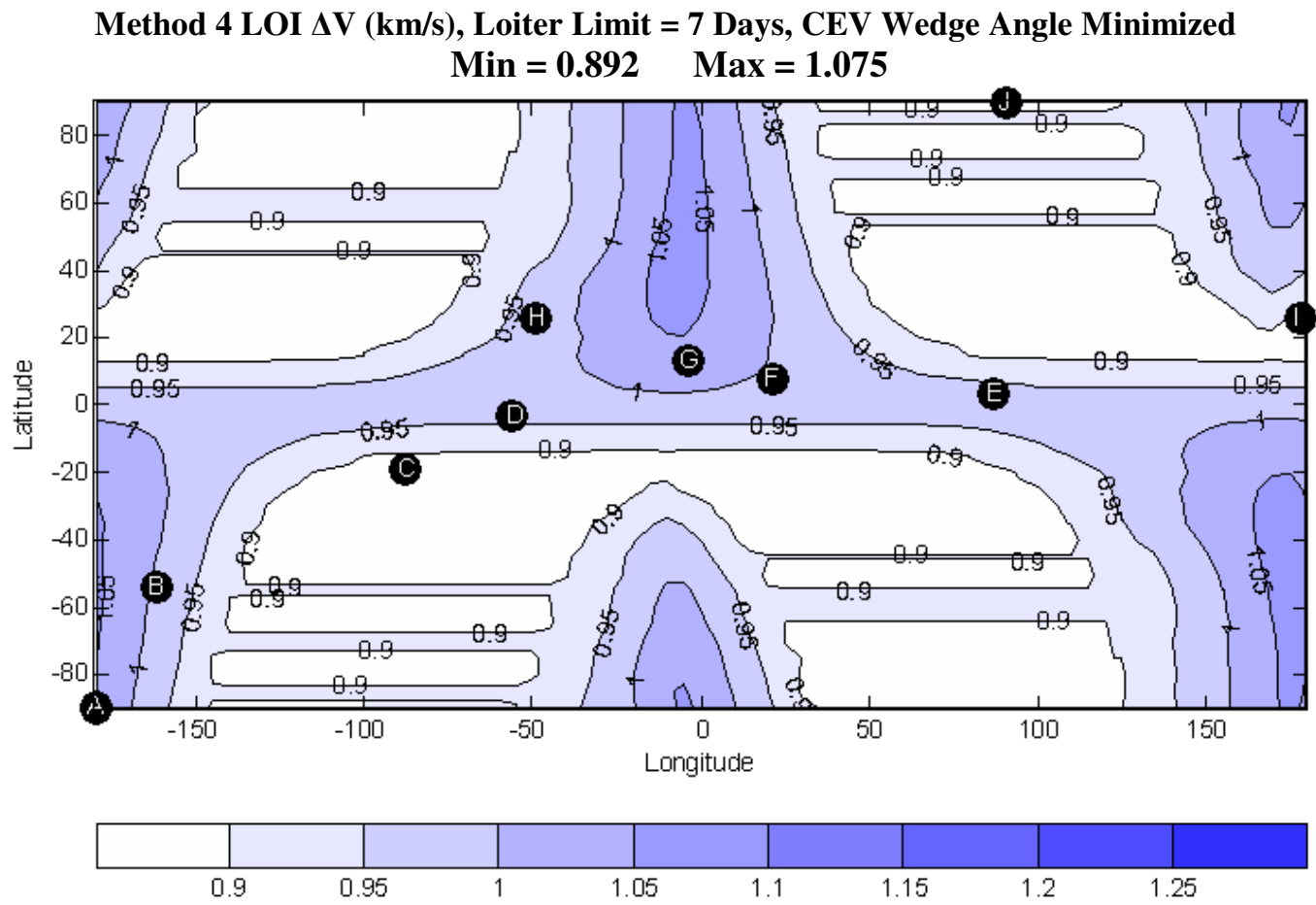

FIGURE 22. Method 4, loiter limit $=7$ days. LLO loiter limit $=7$ days, method 4 , transfer time from the earth to the moon freed, 2nd LOI burn time freed, burn is a pure plane change, earth departure covering the full TLI window, using the worst case Epoch for LOI and using the worst case geocentric wedge angle. 


\section{Method 4 Surface Coverage vs. $\Delta V$ Capability}

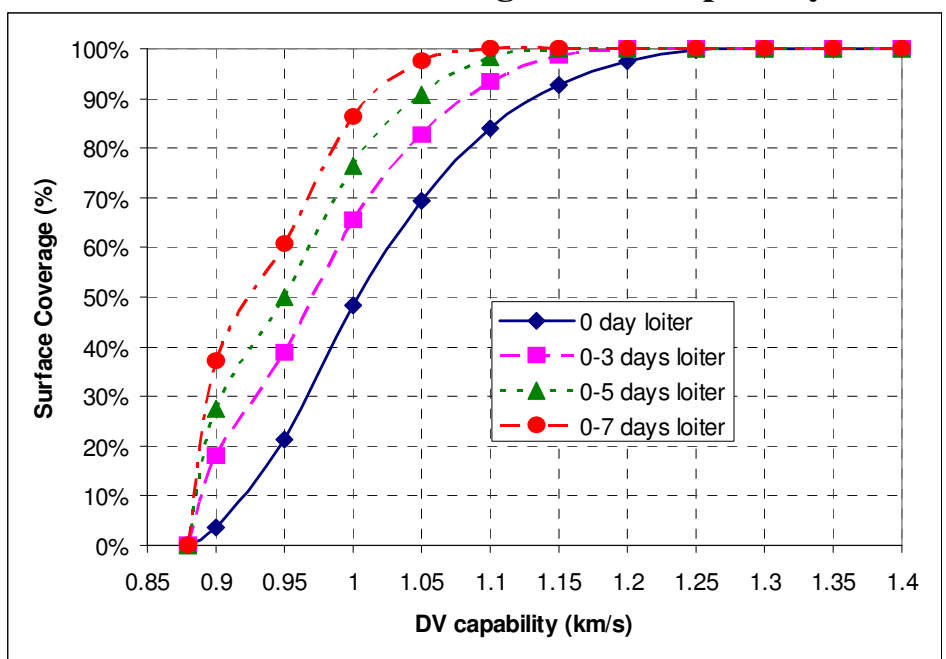

FIGURE 23. Method 4, surface coverage. Percentage of surface coverage for method 4 for different maximum LLO loiter cases.

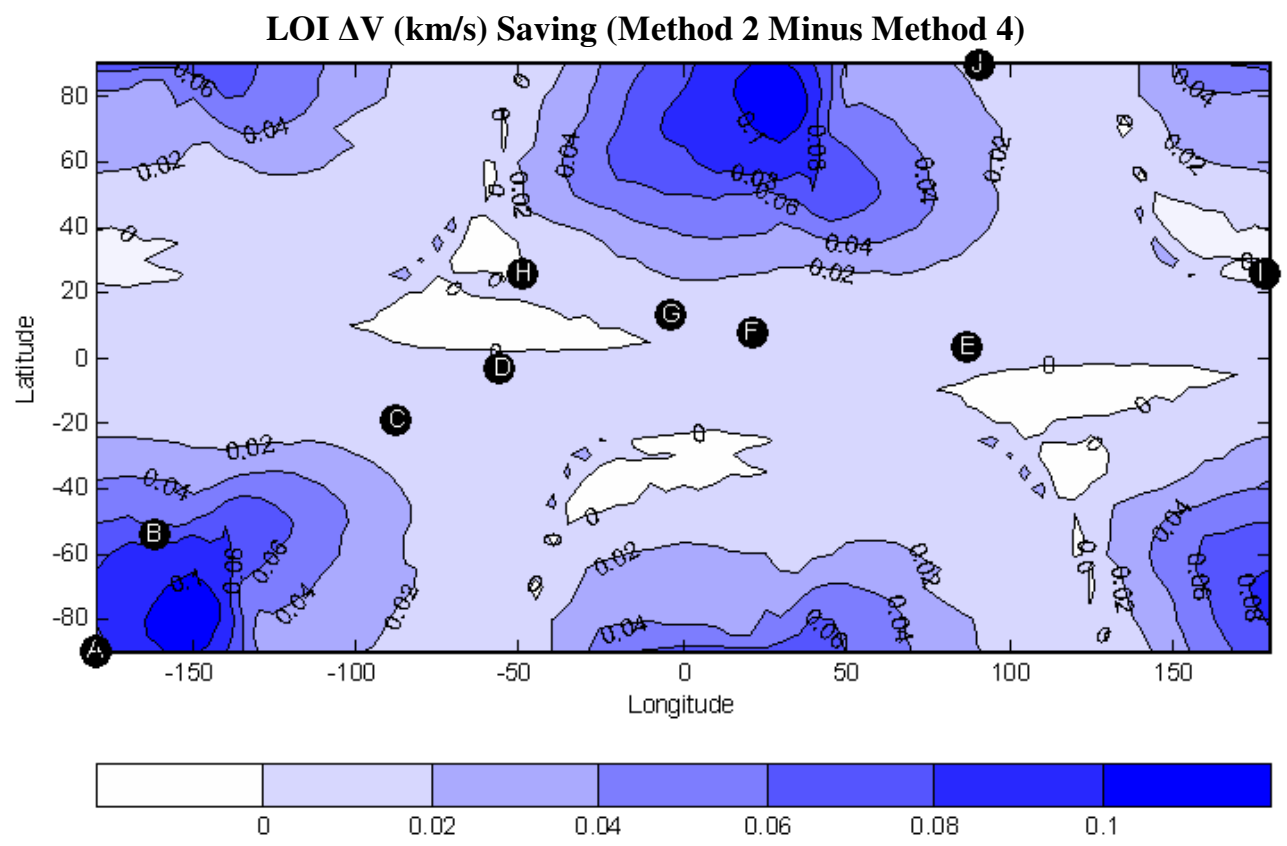

FIGURE 24. Method 2 minus Method 4. No loiter, CEV wedge angle minimized.

\section{Acknowledgments}

The authors would like to thank Jerry Condon, Jacob Williams, Timothy Dawn, Shawn Stuart and David Lee for the technical collaboration and guidance throughout this study. We would also like to thank the NASA Constellation Advanced Program Office for their initiation of this task and for the financial support provided.

\section{References}

${ }^{1}$ Deerwester, J. M., "Plane-Change Requirements Associated with Rendezvous in a Lunar Satellite Orbit," AIAA Journal, Vol. 2, No. 5, 1964, pp. 890-895. 
${ }^{2}$ Condon, G. L., "Lunar Orbit Insertion Targeting and Associated Outbound Mission Design for Lunar Sortie Missions," American Institute of Aeronautics and Astronautics, AIAA Paper 2007-6680, August 2007.

${ }^{3}$ Condon, G. L., Dawn, T., Merriam, R. S., Sostaric, R., and Westhelle, C. H., "CEV Trajectory Design Considerations for Lunar Missions," American Astronautical Society, AAS Paper 07-075, February 2007.

${ }^{4}$ Anon.,"NASA's Exploration Systems Architecture Study: Final Report," NASA TM 2005-214062, November 2005.

${ }^{5}$ Condon, G. L. and Wilson S. W, "Lunar Orbit vs. Libration Point and Lunar Surface Rendezvous Methodologies for Human Lunar Missions," American Astronautical Society, AAS Paper 04-066, 2004.

${ }^{6}$ Eppler, D. B., "Lighting Constraints on Lunar Surface Operations," NASA TM-4271, May 1991.

${ }^{7}$ Ocampo, C, "COPERNICUS: A Trajectory Design and Optimization System,"URL: http://www.ieec.fcr.es/hosted/web-libpoint/abstracts/ocampo.pdf [cited 7 August 2008]. 